\title{
Grundlegende und spezielle Rechtsfragen für autonome Fahrzeuge
}

\author{
Tom Michael Gasser
}

\section{Inhaltsverzeichnis}

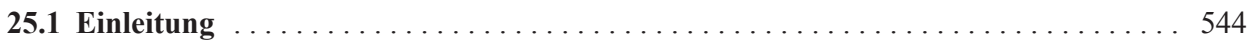

25.2 Bisherige Arbeiten und Vorüberlegungen $\ldots \ldots \ldots \ldots \ldots \ldots \ldots \ldots \ldots \ldots \ldots \ldots \ldots \ldots \ldots \ldots$

25.3 Ausgangssituation des heutigen Straßenverkehrs $\ldots \ldots \ldots \ldots \ldots \ldots \ldots \ldots \ldots$

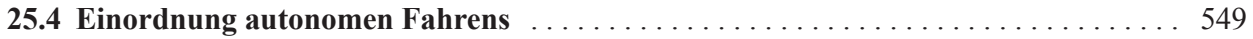

25.4.1 Aktueller Stand marktverfügbarer Fahrerassistenzsysteme . . . . . . . . . . . . 549

25.4.2 Autonomes Fahren . . . . . . . . . . . . . . . . . . . . . . . . 551

25.5 Grundsätzliche Rechtsfragen des autonomen Fahrens $\ldots \ldots \ldots \ldots \ldots \ldots \ldots . \ldots 51$

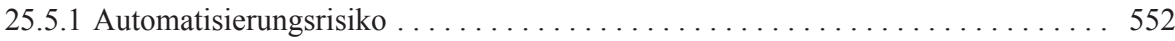

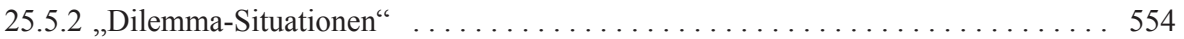

25.5.3 Möglichkeit zur Übersteuerung durch die Passagiere . . . . . . . . . . . . . . . . . 559

25.5.4 Fehlerkompensationsfähigkeit beim autonomen Fahren . . . . . . . . . . 560

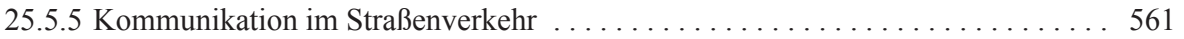

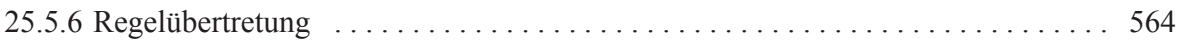

25.6 Spezielle Rechtsfragen autonomen Fahrens $\ldots \ldots \ldots \ldots \ldots \ldots \ldots \ldots \ldots \ldots \ldots$

25.6.1 Ordnungsrechtliche Bewertung fahrerloser Fahrzeuge . . . . . . . . . . . 565

25.6.2 Bewertung autonomen Fahrens nach dem Haftungsrecht im Straßenverkehr . . . 567

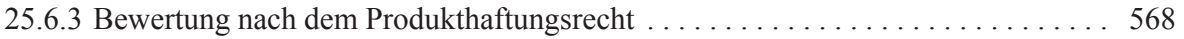

25.6.4 Mögliche Abweichungen der rechtlichen Bewertung im internationalen Kontext . 570

25.6.5 Sonderfrage: Aufsichtspflicht über Insassen autonomer Fahrzeuge . . . . . . . . . 571

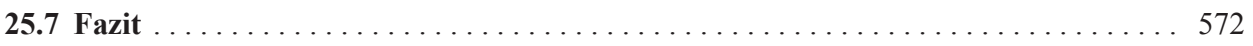

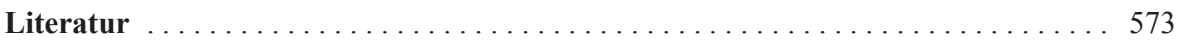

T.M. Gasser $(\bowtie)$

Bundesanstalt für Straßenwesen, Deutschland gasser@bast.de 


\subsection{Einleitung}

Das Projekt „Autonomes Fahren im Straßenverkehr der Zukunft: Projekt Villa Ladenburg“ der Daimler und Benz-Stiftung nimmt Automatisierungsgrade in den Blick, die erst in fernerer Zukunft technisch umsetzbar werden. Die Bearbeitung der aufgeworfenen Rechtsfragen im vorliegenden Kapitel basiert daher wesentlich auf der Beschreibung von UseCases (s. Kap. 2), die erst die für eine Bewertung in einzelnen Punkten notwendige Konkretisierung vornehmen. Unsicherheiten in der Prognose zukünftiger technischer Ausgestaltung sind zu erwarten und werden sich in entsprechendem Maße auf die Annahmen und Schlussfolgerungen dieses Kapitels auswirken. Die resultierende Unsicherheit ist dennoch unvermeidbar, will man wichtige zusammenhängende Fragestellungen voranbringen. Dieses Kapitel versteht sich deshalb vor allem als Diskussionsbeitrag zu den gesellschaftlichen Aspekten automatisierten Fahrens aus einem rechtlichen Blickwinkel und nicht als Rechtsgutachten. Es wird vorwiegend die Situation nach dem in Deutschland aktuell geltenden Recht betrachtet. Die geäußerten Rechtsansichten sind die des Verfassers und beruhen auf neun Jahren Tätigkeit im Bereich der Forschung zu Fahrerassistenzsystemen.

Die im vorliegenden Projekt betrachtete gesellschaftliche Dimension autonomer Fahrzeuge geht nach dem zugrunde gelegten Verständnis weit über die aktuell in Deutschland geforderte Anpassung der rechtlichen Rahmenbedingungen hinaus. Nachfolgend wird eine besondere Fragestellung der ,gesellschaftlichen Akzeptanz“ im Zusammenhang mit den Rechtsfragen von autonomen Fahrzeugen konkretisiert. Sie ist nicht auf den ersten Blick erkennbar und deckt nur einen Ausschnitt des nochmals weiteren Verständnisses im Projekt ab (s. hierzu Kap. 29).

Autonome Fahrzeuge werden sich voraussichtlich nur dann durchsetzen können, wenn ihr gesamtgesellschaftlicher Nutzen den Schaden überwiegt, der mit ihnen einhergeht [1]. Diese frühe, im Zusammenhang mit Fahrerassistenzsystemen aufgestellte These kann in Bezug auf den herausragend wichtigen Belang der Verkehrssicherheit aufgefasst werden. Die Anforderung wäre bereits dann erfüllt, wenn sich die Verkehrssicherheit insgesamt gesehen durch eine entsprechende Weiterentwicklung der Fahrzeuge verbessern würde (was allerdings nicht zwangsläufig gesellschaftliche Akzeptanz zur Folge hat (s. Kap. 29). Hierzu ist bereits heute absehbar, dass autonome, von maschineller Umfeldwahrnehmung abhängig gesteuerte Fahrzeuge nicht völlig fehlerfrei steuern werden. Entsprechend ist zu schlussfolgern, dass es auch im Fall autonomer Fahrzeuge einzelne schwerwiegende Schadensereignisse weiterhin geben dürfte, bei denen im Extremfall mehrere Menschen ihr Leben verlieren werden. Gesellschaftliche Akzeptanz autonomer Fahrzeuge ist deshalb notwendig damit verbunden, dass die Konsequenzen dieser Entwicklung von der Gesellschaft mitgetragen werden. Aus der rechtlichen Perspektive lässt sich zeigen, dass es hierbei ganz grundlegend darum geht, das „Wirken autonomer Fahrzeuge“ im öffentlichen Raum, im Sinne einer eigenständigen maschinellen „Handlung“ ebendort, zu verstehen und zu akzeptieren. Dieser Zustand im Straßenverkehr wäre tatsächlich völlig neuartig (auch andere Verkehrsmittel wie automatisierte, führerlose Bahnen setzen zumeist konsequent den Trennungsgedanken zwischen maschineller Steuerung und öffentlichem Raum mittels 
physischer Barrieren um; es existieren soweit ersichtlich sehr wenige Ausnahmen ${ }^{1}$, die dann aber den Ausschluss resultierender Gefahren nahezu lückenlos über ein Sicherheitskonzept mit einigem Aufwand verfolgen (vgl. [2]).

Dass ein Wirken autonomer Fahrzeuge im öffentlichen Raum des Straßenverkehrs neuartig ist, lässt sich aus rechtlicher Sicht - wie nachfolgend der Versuch unternommen wird - aufzeigen, um deutlich zu machen, dass die gesellschaftliche Akzeptanz einer derart tiefgreifenden Veränderung sich tatsächlich einstellen muss (s. Kap. 30), bevor sie in die Anpassung entsprechender Vorschriften einfließen kann.

\subsection{Bisherige Arbeiten und Vorüberlegungen}

Soweit erkennbar, wurde erstmals anlässlich eines Arbeitstreffens im Oktober 2002 durch Homann [1] die Frage nach gesellschaftlicher Akzeptanz von Fahrerassistenzsystemen mit maschineller Wahrnehmung bearbeitet. Die Inhalte dieser Arbeit lassen sich auf die vorliegende Fragestellung autonomer Fahrzeuge übertragen. Es bleibt lediglich festzuhalten, dass die bereits damals für „Fahrerassistenzsysteme neuer Generation“ diskutierte gesellschaftliche Akzeptanz bislang nicht als „Problem“ im Bewusstsein der Bevölkerung verankert ist, obwohl die seinerzeit diskutierten Systeme inzwischen längst marktverfügbar geworden und hinreichend verbreitet sein dürften, um ein existentes Problem - soweit überhaupt eines besteht - erkennbar werden zu lassen. Dies ist voraussichtlich darauf zurückzuführen, dass bislang spektakuläre negative Effekte durch solche Systeme (wie beispielsweise im Fall von Notbremsassistenz denkbar) nicht verzeichnet werden. Ein Grund hierfür könnte sein, dass die Systeme tatsächlich (nahezu) fehlerfrei arbeiten. Ein differenzierter Erklärungsansatz wird aber sein, dass entsprechende Eingriffe in die Fahrzeugführung nur ausgesprochen kurzfristig und in sehr unfallnahen Situationen erfolgen. So wird technisch eine Auslegung möglich, die ausschließlich dann eingreift, wenn die Auswertung der Umfelderkennung mit sehr hoher Wahrscheinlichkeit ergibt, dass ein Eingriff tatsächlich erforderlich ist - negative Effekte werden so auf ein Minimum begrenzt. Zudem ist zu berücksichtigen, dass der Fahrer weiterhin in der Pflicht bleibt, alle erforderlichen Steuerungshandlungen selbst auszuführen. Dadurch steht der Fahrer potenziell in allen Fällen der Nichtauslösung als umfassende Rückfallebene zur Verfügung. Es ergibt sich somit die untergeordnete Bedeutung solcher Funktionen nicht nur in Bezug auf den zeitlichen Anteil am gesamten Steuerungsgeschehen eines Fahrzeuges, sondern auch hinsichtlich der von ihnen zu erwartenden Zuverlässigkeit. Solche Funktionen können deshalb nur sehr eingeschränkt im Zusammenhang mit eigenständiger bzw. autonomer Fahrzeugsteuerung herangezogen werden: Die untergeordnete Bedeutung und ein gegebenenfalls geringes

1 Projekt RUBIN zur Automatisierung der U-Bahn-Linien U2 und U3 in Nürnberg: Vorübergehend erfolgte der Mischbetrieb von automatisierten und konventionell gesteuerten Zügen auf einer Teilstrecke Rathenauplatz - Rothenburger Straße. Zudem weist das Konzept entlang der Bahnsteige keine physische Trennung, sondern ein sensorbasiertes Bahnsteigsicherungssystem auf. 
„Wirkfeld“ einer Funktion führen zur Stärkung der Fahrerrolle. Darin dürfte ein wichtiger Grund liegen, weshalb die Diskussion um die gesellschaftliche Akzeptanz bislang nicht geführt werden musste.

Es spricht allerdings wenig dafür, dass auch im Fall autonomer Fahrzeuge gesellschaftliche Akzeptanz in diesem Sinne keine Rolle spielen wird: Nimmt man die im vorliegenden Projekt zugrunde gelegten stellvertretenden Applikationen als Grundlage (s. Kap. 2), wird deutlich, dass sie einen sehr hohen Automatisierungsgrad aufweisen. Die ,maschinelle Fahrfähigkeit“" dieser Applikationen als Bezugsgröße wird konsequenterweise hinsichtlich „Fähigkeiten der Perzeption, Kognition, Verhaltensentscheidung sowie Verhaltensausführung“ (s. grundlegende Definitionen in Kap. 2) näher definiert. Es tritt darin eine maschinelle „Autonomie“ des Fahrzeugs zutage, die es erlaubt, vom „Fahrroboter“ „als Subjekt ... analog zur Rolle des Fahrers in heutigen Fahrzeugen“ (s. Kap. 2) zu sprechen. Damit wird aber bereits deutlich, dass es sich hierbei um einen sehr grundlegenden Wandel handelt, der damit einhergehen wird, solche maschinelle Entscheidungen im öffentlichen Raum einzuführen.

Aus rechtlicher Sicht lässt sich zu der Frage gesellschaftlicher Akzeptanz autonomer maschineller Fahrfähigkeiten nur eingeschränkt etwas beitragen: Was rechtliche Vorschriften allerdings in hohem Maße widerspiegeln dürften, ist, was gesellschaftlich als konsensfähig angesehen werden kann - diese Annahme darf jedenfalls dann als gerechtfertigt gelten, wenn einzelne Vorschriften nicht in breiten Kreisen der Öffentlichkeit kritisch diskutiert und hinterfragt werden. Entsprechend lässt sich mittels Gegenüberstellung von autonomen Fahrzeugen mit geltendem Recht darstellen, dass nach dem Sinn und Zweck der Vorschriften gerade nicht eigenständiges „Wirken“ von Maschinen im öffentlichen Raum (verstanden im Sinne von Verhaltensentscheidung und Verhaltensausführung als neuartige Prägung maschinellen Wirkens, s. o.) umfasst ist.

Gesetze sollen abstrakt-generell auf beliebige Lebenssachverhalte anwendbar sein. Diesem Anspruch genügen auch die Vorschriften des Straßenverkehrsrechts grundsätzlich. Wenn allerdings Veränderungen der Lebenswirklichkeit eintreten, die dazu führen, dass vormals zugrunde gelegte Annahmen sich als nicht mehr gültig herausstellen, wie dies durch das Hinzutreten autonomer Fahrzeuge mit Entscheidungswirkung im öffentlichen Raum der Fall sein würde, kann dies über eine Rechtsanwendung nur dargestellt werden. Möglich ist insoweit, die eintretende Veränderung präzise zu beschreiben und die übergeordneten Grundwerte unserer Gesellschaft - wie die Grundrechte - argumentativ heranzuziehen, die den Rahmen beschreiben können, der voraussichtlich die Veränderungen überdauern wird und für die Entwicklung insgesamt maßgeblich ist.

\subsection{Ausgangssituation des heutigen Straßenverkehrs}

Grundlegende staatliche Pflichten lassen sich auch im Bereich von Straßenverkehrsunfällen auf die Verfassung zurückführen. Aufgrund der mit dem Straßenverkehr verbundenen Gefahren stehen vor allem die Grundrechte auf Leben und körperliche Unversehrtheit 
(geschützt durch Art. 2 Abs. 2 Satz 1 Grundgesetz (GG)) im Mittelpunkt. Der sachliche Schutzbereich des Grundrechts auf Leben umfasst den Schutz jedes Menschen (,Jedermanngrundrecht"*) nicht nur vor gezielter Tötung, sondern auch vor Verhaltensweisen, die unbeabsichtigt (ungewollte Eingriffe wie beispielsweise Unfallfolgen) den Tod herbeiführen. Dabei ist die staatliche Schutzpflicht umfassend und gebietet auch Schutz vor rechtswidrigen Eingriffen Dritter. Dies führt in letzter Konsequenz, wenn Eingriffe nicht zu rechtfertigen sind, zur staatlichen Pflicht, ein Verbot solcher Eingriffe - beispielsweise durch den Erlass von Rechtsnormen - sicherzustellen [3].

An dieser Stelle wird eine Diskrepanz zwischen der Lebenswirklichkeit der Gesellschaft und verfassungsrechtlichem Anspruch deutlich. So wird insbesondere darauf hingewiesen, dass ,... die Verkehrssicherheit [kaum diskutiert wird], obwohl die immer noch große Zahl von Verkehrstoten und von dauerhaft schwerbeschädigten Unfallopfern dazu allen Anlass gäbe. “ [3] Auch werden schwere Straßenverkehrsunfälle in den Medien regelmäßig nur als Thema von untergeordnetem, regionalem Interesse behandelt. Das sich darin offenbarende Phänomen des heutigen Straßenverkehrs liegt darin, dass die mit ihm verbundenen Gefahren so wenig Aufmerksamkeit erregen und in der gesellschaftspolitischen Diskussion so wenig Raum einnehmen [4], woraus zumindest ein Indiz für eine breite gesellschaftliche Akzeptanz dieses Zustands abgeleitet werden könnte.

Gleichwohl wirkt sich die Schutzpflicht bezüglich der „Risiken der Technik“ auch im Bereich des Straßenwesens ganz erheblich aus. Eine Statistik der (schwerwiegenden) Straßenverkehrsunfälle wird geführt (gemäß $§ 5$ Abs. 3 Straßenverkehrsunfallstatistikgesetz mit einer Aufgabe der Unfallforschung bei der Bundesanstalt für Straßenwesen), sodass eine präzise Überwachung der Verkehrssicherheitsentwicklung und Unfallursachen erfolgen kann (wobei die Entwicklung der Zahl im Straßenverkehr Getöteter seit vielen Jahren insgesamt gesehen rückläufig ist). Die Verkehrssicherheit ist aber weit darüber hinaus eine Größe von entscheidender Bedeutung im (verkehrspolitischen) Handeln, beispielsweise (aber nicht abschließend) bei dem Erlass und der Änderung von straßenverkehrsrechtlichen (fahrerlaubnisrechtlichen, fahrzeugtechnischen, verhaltensrechtlichen und vieler weiterer) Regelungen, aber auch bei Straßenentwurf, Straßenunterhaltung, der Straßenausstattung etc. Trotz dieser vielfältigen Anstrengungen und laufenden Verbesserungen bleibt der bislang erreichte Stand von 3339 Getöteten und 374.142 Verletzten (in Deutschland im Jahr 2013) [5] eine - zwangsläufig unbefriedigende - Realität.

Letztlich stellt sich aus einer sehr grundlegenden rechtlichen Sicht hierbei die Frage nach der verfassungsrechtlichen Rechtfertigung dieser Eingriffe in das Grundrecht auf Leben und körperliche Unversehrtheit. Sie lassen sich argumentativ über das mit dem Kraftfahrzeugverkehr einhergehende Mobilitätsbedürfnis anderer Grundrechtsträger und deren Bereitschaft, sich dieser Gefahren um des mit ihnen verbundenen Nutzens willen auszusetzen, herleiten. Auch das ist aber nicht allgemein gültig, insbesondere, wenn man berücksichtigt, dass die von Kraftfahrzeugen ausgehenden Gefahren aufgrund der erheblichen Betriebsgefahr im Vergleich mit nichtmotorisierten Verkehrsteilnehmern hier keine völlig widerspruchsfreie Argumentation erlaubt: Während bei motorisierten Verkehrsteilnehmern noch damit argumentiert werden kann, dass sie bereit sind, erhöhte Risiken des 
Kraftfahrzeugverkehrs zu tragen, die sie selbst schaffen, lässt sich dies nicht auf Fußgänger oder Radfahrer übertragen [3]. Fußgänger und Radfahrer haben gleichwohl mit 557 und 354 Getöteten und 30.807 sowie 71.066 der Verletzten insgesamt (für 2013) [5] einen erheblichen Anteil am gesamten Straßenverkehrsunfallgeschehen in Deutschland.

Gleichwohl erscheint es wahrscheinlich, dass in weiten Teilen der Gesellschaft Konsens dahingehend bestehen dürfte, Konsequenzen des Straßenverkehrs angesichts der Bedeutung für die Mobilität der Gesellschaft zu akzeptieren. Wagt man das gedankliche Experiment, weitreichende Einschränkungen des Kraftfahrzeugverkehrs zum Zwecke einer Verbesserung der Verkehrssicherheit in Erwägung zu ziehen, hieße dies zugleich, einige weitere gesellschaftliche Belange außer Acht zu lassen: Unmittelbar wirksame (radikale) Änderungen gingen offensichtlich mit einer erheblichen Einschränkung individueller (motorisierter) Mobilität einher und im Übrigen auch (aber nicht nur) der allgemeinen Handlungsfreiheit (grundrechtlich geschützt über Art. 2 Abs. 1 GG). Weil ein solcher Ansatz extrem wäre, ist seine Verhältnismäßigkeit infrage gestellt: Entsprechende, geeignete Maßnahmen bei einer Fahrleistung auf Straßen von insgesamt 724 Milliarden Kilometern (in 2013, für Deutschland) [5] zu identifizieren, die nicht in einschneidenden Konsequenzen für die wirtschaftliche Entwicklung des Landes, Berufsausübung, öffentliche Daseinsvorsorge und vieles andere mehr münden, erscheinen kaum denkbar. Eine Mehrheitsfähigkeit für Einschränkungen im Bereich des Kraftfahrzeugverkehrs ist vor diesem Hintergrund unwahrscheinlich. Der derzeit praktizierte Ansatz einer kontinuierlichen Verbesserung der Verkehrssicherheit erweist sich darum - wie die bislang insgesamt positive Entwicklung zeigt - als erfolgreich, realistisch und vorzugswürdig. Der oben aus verfassungsrechtlicher Sicht dargestellte Appell, die Verkehrssicherheit stärker zu diskutieren, stellt auch die bestehende rechtliche Situation des Straßenverkehrs nicht infrage, sondern betont zunächst die Bedeutung der Verkehrssicherheitsarbeit in diesem Zusammenhang.

Führt man aber die Ursachensuche für das im Vergleich mit anderen Verkehrsmitteln ungünstige Abschneiden des Straßenverkehrs weiter, lohnt es, auf eine sehr grundlegende Besonderheit von Straßen hinzuweisen: Mit der rechtlichen Einordnung von Straßen als „... öffentliche Sachen im Gemeingebrauch ..., [die] einer unbeschränkten Öffentlichkeit unmittelbar und ohne besondere Zulassung für eine bestimmungsgemäße Benutzung zur Verfügung stehen ...", mithin die Straße als ,, Mehrzweckinstitut “ [6], wird treffend eine Eigenschaft hervorgehoben, in der das Verkehrsgeschehen wie wir es heute kennen - und damit auch das resultierende Unfallgeschehen - wurzelt. Straßen stehen als öffentliche Sache im Gemeingebrauch auch keineswegs nur zum Zweck der Ortsveränderung zur Verfügung (sogenannter Verkehr im engeren Sinne), sondern dienen auch dem geschäftlichen und kommunikativen Verkehr (sogenannter Verkehr im weiteren Sinne) [7]. Es ergibt sich in letzter Konsequenz dieser Zweckbestimmung der „Straße“ eine Vielfalt im Verkehrsgeschehen, verstanden im Sinne einer Vielfalt der möglichen Verkehrsteilnehmer, Verkehrsabläufe, plötzlicher, unerwarteter Ereignisse sowie Zustände und Entwicklungen zwischen Verkehrsteilnehmern auf Straßen. Eine vergleichbare Vielfalt möglicher Interaktionen, an denen nahezu die gesamte Bevölkerung in irgendeiner Form teilhat und das mit einem vergleichbaren Verletzungsrisiko verbunden wäre, ist in anderen Lebensbereichen 
ohne Beispiel. Die Straße hat damit eine vielfältige Funktion, die bei dem von anderen Verkehrsträgern in Anspruch genommenen Raum zumeist nicht besteht. Diese Funktionsvielfalt wirkt sich im Rahmen der in Betracht zu ziehenden Umstände im Zusammenhang mit Fahrzeugführung ganz erheblich aus: Die Anforderungen an die verkehrssichere Durchführung der Fahraufgabe sind komplex und vielfältig. Vom Kraftfahrer sind die umfassende Wahrnehmung der Verkehrssituation und unmittelbare Entscheidung und Umsetzung in geeignete Handlungen, insbesondere die Gefahrerkennung, gefordert. Diese ordnungsrechtliche Aufgabe wird heute im Wesentlichen mit verhaltensrechtlichen und fahrerlaubnisrechtlichen Vorschriften wahrgenommen. Welche Bedeutung die Vielfältigkeit und Komplexität der Aufgabe bei einer Ausführung durch eine maschinelle Fahrzeugsteuerung in rechtlichen Kategorien entfaltet, wird nachfolgend noch herausgearbeitet.

\subsection{Einordnung autonomen Fahrens}

Vor diesen Hintergrund tritt im Rahmen des vorliegenden Projektes zum ,autonomen Fahren" die grundlegende und weitreichende Rechtsfrage, wie autonome Fahrzeuge sich in diese Gemengelage rechtlich einfügen könnten. Als Ansatzpunkt einer Beantwortung ist - wie bereits oben ausgeführt - die beschriebene Eigenschaft solcher Fahrzeuge als „Fahrroboter" bzw. ,Subjekt" analog zum Fahrer (s. Kap. 2) erneut aufzugreifen, auszulegen und hinsichtlich der hiermit verbunden Konsequenzen für die Lebenswirklichkeit darzulegen.

\subsubsection{Aktueller Stand marktverfügbarer Fahrerassistenzsysteme}

Bislang existiert eine Handlungs- und Entscheidungsqualität ausschließlich im Fall menschlicher Handlung (die immer mindestens in Form eigener fahrerischer Wahrnehmung und Entscheidung vorliegt - zumindest im Sinne einer ständigen Verpflichtung hierzu). Tatsächlich handelt es sich hierbei um das heute noch aktuelle Minimum an fahrerischer Beteiligung bei der Fahrzeugsteuerung: Aktuelle Fahrerassistenzsysteme im weiteren Sinn können den Fahrer nur darin unterstützen, das Fahrzeug zu führen, sie ersetzen ihn aber nicht. So ist nach aktuellem Stand (August 2014) eine Arbeitsteilung zwischen Fahrer und Fahrerassistenzsystem denkbar, die aktiv die Längs- und Querführung des Fahrzeuges steuern (auf Basis eigener, maschineller Umfeldwahrnehmung). Diese Steuerung besitzt aber gerade keine eigenständige Entscheidungsqualität: Es wird vielmehr jederzeit vorausgesetzt, dass der Fahrer unmittelbar eingreift und die Steuerung des Fahrzeuges sofort wieder übernimmt, wenn dies erforderlich wird - gleich aus welchen Gründen; beispielsweise könnte auch eine fehlerhafte Umfeldwahrnehmung des Systems zugrunde liegen. Damit liegt aber beim Fahrer eine übergeordnete Rolle und Verantwortung, sodass die Fahrzeugsteuerung durch das System nur abgeleitet und untergeordnet erscheint. Aus technischer Sicht der Arbeitsteilung ist dies derzeit auch notwendig, weil im Markt verfügbare Fahrerassistenzsysteme nicht in der Lage sind, das Erreichen aller Systemgrenzen von 
sich aus zu erkennen. Entsprechend lautet ein charakteristischer Warnhinweis (von mehreren) in der Instruktion für das System „DISTRONIC PLUS“ (eine adaptive Geschwindigkeitsregelung und damit ein System der Längsführung der Firma Daimler AG) nach Stand August 2014:

\section{WARNUNG}

Die DISTRONIC PLUS und der aktive Totwinkel-Assistent sind nur Hilfsmittel, die Sie beim Fahren unterstützen sollen.

Sie können Ihre Aufmerksamkeit nicht ersetzen. Die Verantwortung für den Abstand zu anderen Fahrzeugen, für die gefahrene Geschwindigkeit und für rechtzeitiges Bremsen liegt bei Ihnen. Achten Sie stets auf das Verkehrsgeschehen und Ihre Umgebung. Sie könnten sonst Gefahren zu spät erkennen, einen Unfall verursachen und sich und andere verletzen. [8]

Aus diesem Warnhinweis - der sich auch im Fall anderer heutiger Fahrerassistenzsysteme in vergleichbarer Weise findet - ergibt sich sehr klar, dass das System den Fahrer nur unterstützen kann, während der Fahrer ununterbrochen zu eigener Wahrnehmung der Verkehrssituation (unverändert) angehalten bleibt. Alle durch das System automatisierten Steuerungsvorgänge sind durch den Fahrer zu kontrollieren und gegebenenfalls durch eigene Bedieneingaben zu überstimmen.

Fahrerassistenzsysteme werden darum aus regelungstechnischer Sicht als ,redundantparallele“ Form der Arbeitsteilung von gleichen Aufgaben beschrieben [9]. Diese arbeitswissenschaftliche Beschreibung ist auch vor dem rechtlichen Hintergrund richtig, sagt aber noch nichts darüber aus, wo die Entscheidungsbefugnis im Fall widersprechender Arbeitsausführung liegt. Die Autorität, das Fahrerassistenzsystem jederzeit zu überstimmen, liegt bei Fahrerassistenzsystemen heute immer beim menschlichen Fahrer. Der sich aus den Hinweisen der Bedienungsanleitung (bzw. Instruktion) ergebende bestimmungsgemäße Gebrauch des Systems geht immer dahin, die geeignete Arbeitsausführung des Systems zu beobachten und zu kontrollieren sowie abzuändern, wenn sie in ungeeigneter Weise erfolgt.

Werden die Längs- und die Querführung eines Fahrzeuges, also die beiden wesentlichen Aspekte der Fahraufgabe hinsichtlich der Steuerungsausführung, zeitgleich automatisiert, wird diese Arbeitsteilung heute als teilautomatisch bezeichnet [10]. Dies ändert aber nichts daran, dass auch solche Systeme nicht in der Lage sind, Systemgrenzen von sich aus zu erkennen und deshalb der redundant-parallelen Wahrnehmung, Entscheidung und Handlung eines menschlichen Fahrers (verstanden als dessen Ausübung von Autorität im Sinne einer „Übersteuerung bei jedem in irgendeiner Weise erkennbaren Bedarf") notwendig unterliegen. Somit kommt dem Aspekt der „Autorität“ bei einer durch den Fahrer bestimmungsgemäß zu überwachenden Automatisierung die rechtlich entscheidende Bedeutung zu.

Im Ergebnis bleibt daher festzuhalten, dass den bis heute marktverfügbaren Fahrerassistenzsystemen niemals eine eigenständige, sondern ausschließlich eine abgeleitete Handlungs- und Entscheidungsqualität bei der Fahrzeugsteuerung zukommt, die sich unter vollständiger Autorität des Fahrers vollzieht, der sie ständig bestimmungsgemäß überwacht. 


\subsubsection{Autonomes Fahren}

Grundlegend anders stellt sich dies im Fall autonomen Fahrens dar, das mit vier stellvertretenden Applikationen für die vorliegende Bewertung (s. Kap. 2) herangezogen wird. Alle vier Applikationen sehen gleichermaßen einen Fahrroboter vor, der die Fahrzeugsteuerung übernimmt. Selbst die Applikation des ,Autobahnautomaten mit Verfügbarkeitsfahrer Autobahnpilot", die deutlich an heutige Fahrerassistenzsysteme erinnert, setzt gerade nicht voraus, dass der „Fahrer“ während der automatisierten Fahrt überhaupt noch eine Aufgabe wahrnimmt, die dem heutigen Bild eines „Fahrers“ entspricht. Dies tritt in der eindeutigen Formulierung zutage, der Fahrer werde „,... während der autonomen Fahrt zum einfachen Passagier ...".

Das autonome Fahren sieht damit im Kern vor, dass die redundante Parallelität in der zeitgleichen Aufgabenwahrnehmung durch Fahrer und System zugunsten einer - gegebenenfalls zeitlich und räumlich begrenzten - eigenständigen maschinellen Fahrzeugsteuerung entfällt. Die zutreffend daher nur noch als „Fahrzeugnutzer“ bezeichnete Person hat zwar im Fall der o.g. Applikation „Autobahnpilot“ eine als „,dominant“ ausgewiesene Eingriffsmöglichkeit in die Fahrzeugsteuerung, die sich grundsätzlich nicht von der in Abschn. 25.4.1 beschriebenen ,Autorität“" im Fall von Fahrerassistenzsystemen unterscheidet. Allerdings fehlt es mit einiger Wahrscheinlichkeit an der Grundlage für ihre Ausübung: Sobald sich die Rolle des Fahrers derart verändert, dass nicht nur die Handlungsausführung, sondern auch die eigene Beobachtung der Verkehrssituation und die hierauf beruhende Bewertung maschineller Steuerungsentscheidungen entfällt, wird auch zwangsläufig die Bedeutung dieser „Dominanz“ oder „Autorität“ eingeschränkt sein: Es fehlt auch bei Anwesenheit des Fahrzeugnutzers die Situationswahrnehmung und damit de facto an einer Grundlage für die Ausübung von „Dominanz“ bzw. „Autorität““. Im Fall der beiden Applikationen, die sich durch die räumliche Abwesenheit des Fahrzeugnutzers auszeichnen (s. die stellvertretenden Applikationen „Autonomes Valet-Parken“ und „Vehicle-onDemand“ in Kap. 2) tritt das Fehlen jeglicher Grundlage für die Ausübung von „Dominanz“ bzw. „Autorität“" noch offensichtlicher zutage.

Das Fehlen einer Grundlage für eine unmittelbare Beobachtung von Verkehrssituationen als Ausgangspunkt einer Fahrzeugsteuerung erlaubt nur den Rückschluss auf eine Eigenständigkeit bzw. „Autonomie“ der maschinellen Steuerung. Die insoweit alles entscheidende Bedeutung der Autonomie maschineller Fahrzeugsteuerung im vorliegenden Zusammenhang hat daher konsequenterweise als adverbiale Bestimmung auch Eingang in den Namen des zugrundeliegenden Projektes gefunden.

\subsection{Grundsätzliche Rechtsfragen des autonomen Fahrens}

Rechtsfragen im Zusammenhang mit dem autonomen Fahren lassen sich, wie bereits in Abschn. 25.2 ausgeführt, nicht anhand der heutigen Rechtsgrundlagen zufriedenstellend oder erschöpfend beantworten. Dies begründet sich daraus, dass die Rechtsgrundlagen des 
Straßenverkehrsrechts bei ihrer Entstehung und Weiterentwicklung bislang nur Erwägungen zugrunde legen konnten, die zum Regelungszeitpunkt regelungsbedürftig waren. Im Bereich des öffentlichen Straßenverkehrs hat sich die Frage autonomer Fahrzeuge bislang nicht gestellt - auch nicht angesichts heute marktverfügbarer Fahrerassistenzsysteme (s. Abschn. 25.4.1): Es war bislang immer davon auszugehen, dass ein Fahrer die Fahrzeugsteuerung mindestens im Sinne der redundant-parallelen Aufgabenwahrnehmung noch ausführt. Zieht man demgegenüber autonomes Fahren in Betracht, liegt darin ein Wechsel von grundlegender Bedeutung hin zur eigenständigen maschinellen Fahrzeugsteuerung.

\subsubsection{Automatisierungsrisiko}

Für autonome Fahrzeuge wird angenommen, dass „Hardware-Ausfälle und SoftwareFehler ... auch bei autonom fahrenden Fahrzeugen auftreten [können]“, wobei diese „,nach aktuellem Stand der Technik“ entwickelten Fahrzeuge als „... mindestens so zuverlässig und sicher ... wie es heutige Fahrzeuge sind" eingestuft werden. Im Weiteren wird aber zugleich deutlich, dass diesbezüglich noch erhebliche Unsicherheit herrscht, da die „Erfolgsquote“ bei der Fahrzeugführung als ,ähnlich der menschlichen Qualität und Erfolgsquote" angenommen wird, dies aber derzeit eine vorsichtige erste Einschätzung von Experten darstellt, die nur als Diskussionsgrundlage für das vorliegende Projekt dient (s. Kap. 2). Tatsächlich kann also die Leistung maschineller Fahrzeugsteuerung heute noch nicht abschließend beurteilt werden; anzunehmen ist aber, dass einerseits ein Automatisierungsrisiko verbleiben wird, dieses andererseits jedoch nicht höher liegt als das resultierende Risiko menschlicher Fahrzeugführung.

\subsubsection{Das Automatisierungsrisiko vor dem Hintergrund der Grundrechte}

Nimmt man dieses somit für möglich gehaltene Automatisierungsrisiko in den Blick und zieht die Situation bezogen auf das Grundrecht auf Leben und körperliche Unversehrtheit im heutigen Straßenverkehr mit heran (s. Abschn. 25.3), wird deutlich, dass der Wandel von menschlich kontrollierter zu eigenständiger, maschineller Fahrzeugsteuerung als ",wesentlich für die Verwirklichung der Grundrechte" angesehen werden dürfte [11]. Aufgrund des erheblichen Eingriffs, den ein solches, neuartiges Automatisierungsrisiko in das Grundrecht auf Leben und körperliche Unversehrtheit bedeuten würde, ist es als wahrscheinlich anzusehen, dass eine Entscheidung über die Ermöglichung autonomer Fahrzeuge und damit über ein eigenständiges maschinelles Automatisierungsrisiko im Straßenverkehr dem Gesetzgeber obliegt. Der Gesetzgeber wäre verpflichtet, die wesentlichen Entscheidungen selbst zu treffen, was sich aus dem Demokratieprinzip und Rechtsstaatsgebot ergibt. Die für die Grundrechtsverwirklichung maßgeblichen Regelungen dürften somit nicht dem Handeln und der Entscheidungsmacht der Exekutive überlassen werden [12]. Diese Argumentation stellt die Neuartigkeit eines solchen Automatisierungsrisikos in den Mittelpunkt, weil hiermit die Fahrzeugsteuerung insgesamt revolutioniert würde. Zugleich ist aber einschränkend festzustellen, dass die realistisch zu erwartende Einführung automa- 
tisierter Fahrzeugführung allmählich über die immer weitere Verbesserung bereits heute verfügbarer Fahrerassistenzsysteme ,, stufenweise“ (s. Kap. 2) erfolgt. Damit wird die Frage aufgeworfen, ob der Wandel hin zu maschineller Fahrzeugsteuerung zum Zeitpunkt der Entscheidung hierüber noch als „,wesentlich“ anzusehen sein wird. Das Bundesverfassungsgericht ist mit der Anwendung des Parlamentsvorbehaltes im Rahmen der Grundrechte auf Leben und körperliche Unversehrtheit in der Vergangenheit eher zurückhaltend gewesen und hat es genügen lassen, dass ein fraglicher Typus an Lebensrisiko (im konkreten Fall war die „Brüter-Technologie“ Gegenstand) von dem im Atomgesetz zum Ausdruck kommenden gesetzgeberischen Willen umfasst ist [3], [13]. Auch ist nicht erforderlich, dass die Risiken und Folgen des Straßenverkehrs als solche explizit benannt werden, die die Grundrechte auf Leben und körperliche Unversehrtheit einschränken (sodass sich allein hieraus auch heute keine Verfassungswidrigkeit des Straßenverkehrsgesetzes ableitet) [13]. Es bleibt deshalb die Frage, ob das Straßenverkehrsgesetz in der heutigen Form die neuartige maschinelle Steuerungsqualität umfassen kann. Zum gegenwärtigen Zeitpunkt (Stand: August 2014) begründet die Neuartigkeit und die Eigenständigkeit der maschinellen Entscheidungsqualität hieran erheblichen Zweifel.

Stellen sich die zugrunde gelegten Annahmen und Bezüge als richtig heraus, wäre die Frage der Ermöglichung eines maschinellen Steuerungsrisikos im öffentlichen Straßenverkehr durch ein formelles Gesetz zu regeln (sogenannter Vorbehalt des Gesetzes). Dem Gesetzgeber stünde es dabei frei, Risiken zuzulassen, die „,unterhalb der Gefahrenschwelle“ bleiben und sich deshalb - auch angesichts der Freiheitsausübung des Risikoverursachers - rechtfertigen lassen. Er ist indes nicht hierauf beschränkt und kann auch zur Risikovorsorge unterhalb der Gefahrenschwelle tätig werden, soweit dies noch als verfassungsrechtlich verhältnismäßig anzusehen ist [3]. Hinsichtlich des Ausmaßes eines möglichen Automatisierungsrisikos erscheint deshalb - schon aufgrund der Indizwirkung der Situation im heutigen Straßenverkehr - eine Sicherheit und damit auch Regelungsbefugnis des Gesetzgebers durchaus realistisch, die sich im Bereich bewegt, der „mindestens so zuverlässig und sicher ist, wie es heutige Fahrzeuge sind“" (s. Kap. 2).

\subsubsection{Haftung des Produktherstellers für die eigenständig wirkende automatisierte Fahrzeugsteuerung?}

Die Produkthaftung des Herstellers bei vollautomatisierten Systemen (zur Einordnung der vorliegenden Use-Cases als „,vollautomatisiert“ s. Kap. 2) könnte wesentlich durch den seitens des Herstellers intendierten Gebrauch des Produktes bestimmt werden. Soweit Systemfunktionen - wie im Fall der Vollautomatisierung - keine notwendige Rolle des Fahrers bei der Fahrzeugsteuerung mehr vorsehen, könnte sich im Rückschluss ergeben, dass die Annahme eines Anscheinsbeweises im Fall eines Unfallschadens solcher Fahrzeuge naheliegt: Tritt während einer maschinell gesteuerten Fahrt ein (Unfall-)Schaden auf, stellt sich die Frage, ob dies auf einen kausal zugrunde liegenden Produktfehler schließen lässt (soweit die relevante Ursache dieses Schadens nicht in einem Eingreifen des Fahrers in Form einer Übersteuerungshandlung oder einem allein ursächlichen Fehlverhalten eines anderen Verkehrsteilnehmers liegt - alles im Rahmen der geltenden Darlegungs- und 
Beweislast in Zivilverfahren) [10]. Entscheidend wäre im Ergebnis, ob eine fehlerhafte maschinelle Steuerungsentscheidung letztlich als Produktfehler einzuordnen ist und es so in praktisch allen Fällen fehlerhafter Steuerung zur Annahme einer zivilrechtlichen Haftung des Herstellers kommen könnte.

Damit würden - neben dem Fahrzeughalter (vgl. dazu [10]) - letztlich (fast) immer die Hersteller das zivilrechtliche Haftungsrisiko für das mit maschinellem Wirken verbundene Automatisierungsrisiko tragen. Angesichts der erweiterten Einflussmöglichkeiten einer maschinellen Fahrzeugsteuerung (s. hierzu vertiefend Abschn. 25.5.2) würde sich der Anwendungsbereich für steuerungsrelevante Fehler gegenüber einem Fahrer sogar noch ausweiten.

Hier ist allerdings kritisch zu hinterfragen, ob dieser Rückschluss richtig ist: Die Argumentation folgt in weiten Teilen der Annahme, dass die Ursache von Unfällen heute regelmäßig auf fehlerhafte fahrerische Steuerungsentscheidungen zurückzuführen ist. Die (zukünftig gegebenenfalls maschinelle) Fahrzeugsteuerung stellt aber möglicherweise nur eine von mehreren relevanten Unfallursachen dar.

Das heutige Straßenverkehrsrecht scheint noch immer durch ein anderes Grundverständnis geprägt. Deutlich wird dies etwa in der aktuellen Fassung des Straßenverkehrsgesetzes, das von der Existenz ,unabwendbarer Ereignisse“ ausgeht (diese sind heute in $\S 17$ Abs. 3 Straßenverkehrsgesetz bei der Zuordnung von Schadensverursachungsanteilen zwischen Kraftfahrzeugen weiterhin von Bedeutung). Dabei ist aber hervorzuheben, dass die heute hierunter zu fassenden Fälle im streng naturwissenschaftlichen Sinn und unter Berücksichtigung des heutigen Standes der Technik voraussichtlich nur teilweise als „unabwendbar“ zu qualifizieren sein werden. Dennoch wird das Verständnis dieses juristischen Begriffs bis heute nur insoweit eingeschränkt, als ,äußerste mögliche Sorgfalt“ und das „Verhalten eines ,Idealfahrers“" im Sinne durchschnittlicher Verhaltensanforderungen (und in Abgrenzung zum gedachten „Superfahrer") hierfür genügen [14]. (Erkennbar liegt hier aber ein anderer Gedanke zugrunde: die Frage nach dem Verschulden eines Unfalls, nicht naturwissenschaftliche Kausalität seines Auftretens.)

Die konsequente Anwendung der Frage nach naturwissenschaftlicher Kausalität könnte bedeuten, dass gegebenenfalls nur eine Teilmenge von Schäden, die während automatisierter Fahrzeugsteuerung auftreten, tatsächlich auf eine fehlerhafte Steuerung (oder einen sonstigen Produktfehler) zurückzuführen sein wird. Dies würde im Ergebnis den oben dargestellten Rückschluss von einem Schaden auf das Vorliegen eines Produktfehlers während automatisierter Steuerung grundlegend infrage stellen. Die offene Frage in diesem Zusammenhang lautet deshalb, inwieweit das heutige „Verkehrssystem Straße“ eine eigenständig relevante Ursache für Unfallschäden (gegenüber gegebenenfalls maschinellen Steuerungsentscheidungen) darstellt.

\subsection{2 „Dilemma-Situationen“}

Der einprägsame Begriff der „Dilemma-Situation“ beinhaltet im Rahmen der rechtlichen Diskussion zunächst gleich zwei aufeinander aufbauende relevante Aspekte, die charakte- 
ristische Merkmale eines maschinellen Wirkens pointiert beschreiben und für den Extremfall die Konsequenzen eines solchen Wandels mit einmaliger Klarheit aufzeigen können: Diese sind erstens die Erweiterung der Einflussmöglichkeit von Fahrzeugsteuerung in zeitkritischen Situationen und zweitens die Frage nach der Umsetzung einer maschinellen Steuerungsentscheidung im grundrechtsrelevanten Bereich.

Zunächst muss grundlegend die Existenz von „Dilemma-Situationen“ im Straßenverkehr infrage gestellt werden. Unklar erscheint insbesondere, ob das zugrunde liegende gedankliche Modell anderweitiger Alternativlosigkeit trägt: Im Straßenverkehr treten im Einzelfall vielfältige, nacheinander geschaltete und stark situationsabhängige Einflussmöglichkeiten bei der Fahrzeugsteuerung auf. Vorangehende alternative Steuerungsentscheidungen im Straßenverkehr bieten somit - möglicherweise, dies wäre näher zu untersuchen - die Möglichkeit, auf das Zustandekommen einer alternativlosen Situation, die zwangsläufig zu einem Schaden führt, Einfluss zu nehmen. Es erscheint nicht ausgeschlossen, durch ein vorausschauendes Steuerungsverhalten alternativlose Situationen von vornherein zu vermeiden. Umgekehrt kann sich aber auch ergeben, dass bestimmte Gefahren des Straßenverkehrs auf seine prägenden Merkmale zurückzuführen und nicht vermeidbar sind (beispielsweise auf die Vielfalt möglicher Interaktion von unterschiedlich geschützten Verkehrsteilnehmern). Dann ist im Ausnahmefall aber auch die Koinzidenz von zwei möglichen Schädigungen denkbar und die Befassung mit „Dilemma-Situationen“ notwendig. In juristischen Kategorien wird hier die - naturwissenschaftlich zu bestimmende - Frage nach relevanten Unfallursachen sowohl hinsichtlich interner (fahrzeugsteuerungsabhängiger) als auch externer (verkehrssystemabhängiger) Faktoren kritisch hinterfragt.

Unabhängig vom Ergebnis dieser theoretischen Betrachtung von Fahrzeugsteuerung im Straßenverkehr ergeben sich in rechtlicher Hinsicht diskussionswürdige Aspekte: So kann anhand von „Dilemma-Situationen“ der grundrechtliche Rahmen für maschinelle Steuerungsentscheidungen autonomer Fahrzeuge aufgezeigt werden. Legt man die Existenz von „Dilemma-Situationen“ zugrunde, ergibt sich darüber hinaus, dass eine Beschränkung der Herstellerverantwortung in diesen Fällen geboten sein könnte, weil Schädigungen sich dann durch Steuerungshandlungen als ebenso unvermeidbar erweisen würden, wie sie es im Fall der Existenz eigenständiger Risiken des „Verkehrssystems Straße“wären (s. Abschn. 25.5.1).

Schließlich ist darauf hinzuweisen, dass „Dilemma-Situationen“ auch der Diskussion ethischer Aspekte in verschiedenen Ausprägungen zugrunde gelegt werden, wobei sie auch dort der Pointierung und damit Bearbeitung der übergeordneten ethischen Fragestellung dienen (s. Kap. 4).

\subsubsection{Erweiterung der Einflussmöglichkeit}

Das gedankliche Modell der „Dilemma-Situation“ für autonome Fahrzeuge legt zunächst als Arbeitshypothese zugrunde, dass bestimmte, gesteigert unfallnahe Situationen durch den Einsatz maschineller Fahrzeugsteuerung noch beeinflusst werden könnten: In vielen Fällen könnte es dadurch möglich sein, heute als konkret gefährdet anzusehende Rechtsgüter in buchstäblich letzter Sekunde zu ,retten“. Diese Situationen bedürfen nach heutigem Stand der Technik (bei allein menschlicher Fahrzeugsteuerung) immer der Berück- 
sichtigung einer Reaktionszeit des Fahrers [14]. Die hierdurch verzögerte Steuerungshandlung kann einzelfallabhängig das Auftreten eines Unfalls oder seine Folgen beeinflussen. Tatsächlich erscheint diese Arbeitshypothese im Zusammenhang mit einem Wandel hin zu maschinellen Steuerungsentscheidungen keineswegs abwegig. Es ließe sich zudem noch annehmen, dass ein weiterer Vorteil dadurch erreicht werden kann, dass alternative Steuerungsmöglichkeiten (wie beispielsweise das Ausweichen anstelle des Bremsens vor einem konkret gefährdeten Fußgänger, was dem Durchschnittsfahrer selten gelingt [15]) von einer maschinellen Steuerung mitberücksichtigt werden können. Eine wesentliche, den „Dilemma-Situationen“ zugrunde liegende Arbeitshypothese lautet deshalb, dass die maschinelle Fahrzeugsteuerung potenziell zur Kontrolle von bislang nicht oder nur verzögert beherrschten Verkehrssituationen führt.

\subsubsection{Die automatisierte Steuerungsentscheidung}

Die hierauf aufbauende weitere Annahme im gedanklichen Modell der „Dilemma-Situation“ berücksichtigt die neuartige maschinelle „Entscheidungsqualität“ einer autonomen Fahrzeugsteuerung. Diese Sichtweise kommt ausdrücklich in der Bezeichnung des „Fahrroboters“ als ,, Subjekt “zum Ausdruck, die sich - mit aller in dieser Hinsicht gebotenen Vorsicht - in den grundlegenden Definitionen des vorliegenden Projektes äußert (s. Kap. 2). Um diesen Effekt pointiert hervorzuheben und die ethische Dimension einer maschinellen Steuerungsentscheidung zu argumentieren, erfolgt unter Hinzunahme eines Entscheidungsdilemmas die - in der Natur eines Dilemmas liegende anderweitig ausweglose - Zuspitzung. Eine solche Zuspitzung ist nicht als völlig unrealistisch anzusehen und schon deshalb unbedingt diskussionswürdig, weil es um die gesellschaftliche Akzeptanz einer weitreichenden maschinellen Entscheidungsqualität geht, die hierdurch in den Mittelpunkt gerückt wird. Diese maschinelle Entscheidung kann im Einzelfall - wie in den „DilemmaSituationen“ konstruiert - das Recht des (konkret gefährdeten) Einzelnen auf Leben und körperliche Unversehrtheit betreffen und liegt insoweit grundsätzlich nicht anders als im Fall der grundrechtsrelevanten Gefahren im heutigen Straßenverkehr (s. hierzu Abschn. 25.3). Der wesentliche Unterschied liegt einzig in der zugrunde liegenden maschinellen Steuerung.

\subsubsection{Kritische Analyse der "Dilemma-Situation"}

Man wird bei einer theoretischen Aufarbeitung möglicher Konsequenzen maschineller Steuerungsentscheidungen der Sache nur gerecht, wenn auch darauf hingewiesen wird, dass die „Dilemma-Situation“ in Gestalt des „Entscheidungsdilemmas“, soweit es überhaupt existiert, die absolute Ausnahme darstellen wird. Für eine sachliche Einordnung ist deshalb einleitend darauf hinzuweisen, dass es sich von vornherein nur um eine sehr seltene Ausnahmesituation handeln kann.

Die dem Dilemma zugrunde liegende Annahme ist, dass keine Alternative zur Schädigung von zwei im Wesentlichen gleichrangigen Rechtsgütern im konkreten Einzelfall denkbar ist, obwohl die maschinelle Fahrzeugsteuerung alle alternativ möglichen Steuerungsentscheidungen berücksichtigt hat. Wenn aber in der konkreten Situation - unter 
Beachtung des Steuerungsverhaltens vor Konkretisierung der Gefahr - keine Alternative zur Schädigung verbliebe, können gleichwohl relevante alternative Ursachen als das Steuerungsverhalten in der vorgelagerten Kausalkette in Betracht gezogen werden. So erscheint es etwa plausibel anzunehmen, dass Gefahren möglicherweise nicht allein in der Fahrzeugsteuerung liegen, sondern dem Straßenverkehr als solchem innewohnen können und somit bereits auf die in Abschn. 25.3 beschriebene Komplexität und Vielfalt möglicher Situationen im „Verkehrssystem Straße“ zurückzuführen sind. Dies erscheint insbesondere dann wahrscheinlich, wenn man beispielsweise den Einfluss der Fahrgeschwindigkeit berücksichtigt, deren Höhe voraussichtlich in den meisten Fällen eine notwendige Ursache (im naturwissenschaftlichen Sinn) bei dem Zustandekommen von Verkehrsunfällen darstellen wird. Die „normale“ Fahrgeschwindigkeit ist aber zugleich Bestandteil des heutigen Verständnisses vom „Verkehrssystem Straße“ und bestimmt in weiten Teilen sein Gepräge. Das Bild vom heutigen Straßenverkehr erscheint im Einzelfall durchaus risikobehaftet: So ist durchaus zu hinterfragen, ob in einzelnen Situationen - wie beispielsweise im Fall der Vorbeifahrt an einem Fußgänger - ein Risiko liegt [16], das angepasst werden könnte, um bestehenden Unfallrisiken im Straßenverkehr zu begegnen.

\subsubsection{4 „Dilemma-Situationen“ vor dem Hintergrund der Grundrechte}

Die rechtliche Bewertung von „Dilemma-Situationen“ sollte konsequent in den übergeordneten rechtlichen Rahmenbedingungen, den Grundrechten, ihren Ausgangspunkt nehmen. Im Bereich des Grundrechtes auf Leben und körperliche Unversehrtheit ändert sich durch den Wandel von menschlicher Fahrzeugsteuerung zu maschinellem Wirken nichts von grundlegender Bedeutung: Es kommt insbesondere nicht zu einem „gezielten“ Eingriff in das Leben oder die körperliche Unversehrtheit, der aufgrund der Bedeutung dieser Rechtsgüter als Höchstwert bzw. ihrer fundamentalen Bedeutung praktisch nicht zu rechtfertigen wäre [3]. Zwar ist, wie noch im Zusammenhang mit der Produkthaftung zu diskutieren sein wird, jede Steuerungsentscheidung durch die Programmierung des entsprechenden Systems unter bestimmten Randbedingungen vorgegeben und somit letztlich nicht zufällig, allerdings handelt es sich dabei gerade nicht um die Konkretisierung eines bestimmten Handlungsablaufes. Die Programmierung einer autonomen Fahrfunktion gibt vielmehr (nur) vor, welche Gesichtspunkte zu berücksichtigen sind, sodass hieraus unter mehreren Alternativen diejenige gewählt werden kann, die einen Schaden nach Möglichkeit ganz vermeidet oder den geringsten Schaden verursacht. Gerade in dieser situationsabhängigen Berücksichtigung von Handlungsalternativen liegt der entscheidende Mehrwert einer maschinellen Fahrzeugsteuerung, die in Abschn. 25.5.2.1 als ,Erweiterung der Einflussmöglichkeit" durch maschinelle Steuerung beschrieben wurde. Damit werden aber im Rahmen der Programmierung keine Steuerungsentscheidungen getroffen, sondern (nur) abstrakte Kriterien für die einzelfallbezogene Steuerungsentscheidung vorgegeben. Hieraus wird deutlich, dass es sich letztlich weiterhin um ein abstraktes Risiko handelt, das eine maschinelle Steuerung im Straßenverkehr bedeutet. Sie wäre deshalb vor dem Hintergrund der Grundrechte nicht anders zu behandeln als eine menschliche Fahrzeugsteuerung auch, die die bereits in Abschn. 25.3 dargestellten Risiken birgt. Somit sind die hiermit einherge- 
henden unbeabsichtigten Schutzgutverletzungen (vgl. [3]), die Unfälle unter maschineller Fahrzeugsteuerung im Straßenverkehr ebenso herbeiführen, wenn man vom Vorliegen eines Automatisierungsrisikos ausgeht (s. Abschn. 25.5.1), vor dem Hintergrund des Grundrechtes auf Leben und körperliche Unversehrtheit nicht anders zu beurteilen als heutige Risiken im Straßenverkehr auch.

Ein Gesichtspunkt sollte aus grundrechtlicher Sicht allerdings besondere Berücksichtigung finden: Soweit sich ergibt, dass die maschinelle Umfelderkennung ausreicht, um nichtmotorisierte Verkehrsteilnehmer (Fußgänger und Radfahrer) als solche zu erkennen, wäre ihr Schutz im Rahmen sich ergebender Handlungsvarianten besonders zu berücksichtigen (s. Argumentation in Abschn. 25.3).

Das mit dem Begriff der „Dilemma-Situation“ beschriebene Entscheidungsdilemma zwischen zwei gleichwertigen Rechtsgütern lässt sich indes vor dem Hintergrund des Grundrechts auf Leben und körperliche Unversehrtheit nicht auflösen: Eine Abwägung mit dem gleichwertigen und im Fall des Lebens als verfassungsrechtlicher „Höchstwert“ geschütztes [3] Grundrecht anderer Grundrechtsträger hat zu unterbleiben und ist unzulässig. Aus rechtlicher Sicht kann deshalb - geht man von der Existenz solcher „Dilemma-Situationen“ in der Realität aus - kein Beitrag im Sinne einer Entscheidung ausgehend vom heutigem Stand der Grundrechtsdogmatik erfolgen, vielmehr würde eine lösungsbedürftige, neuartige Frage aufgeworfen. Von Bedeutung wäre darum, die Frage danach zu klären, ob „Dilemma-Situationen“ tatsächlich in dieser Weise auftreten, also insbesondere, ob die relevante Ursache ihrer Entstehung tatsächlich auf die maschinelle Steuerungsentscheidung zurückzuführen ist und nicht auf ein inhärentes Risiko des „Verkehrssystems Straße“ - beispielsweise aufgrund der dort gefahrenen Geschwindigkeiten in bestimmten Situationen (s. hierzu bereits Abschn. 25.5.2.3). Sollte sich ergeben, dass „Dilemma-Situationen“ existieren und in maschineller Steuerung ihre relevante Ursache finden, würde es sich um eine Frage handeln, die transparent gemacht und - gesellschaftliche Akzeptanz eines Automatisierungsrisikos vorausgesetzt - diskutiert werden müsste. Notwendig wäre dann voraussichtlich die Erstellung eines Kataloges mit anerkannten Entscheidungskriterien für solche Situationen.

Bei alledem wäre zu berücksichtigen, dass die Frage erst durch die Erweiterung der Einflussmöglichkeit aufkommt (s. Abschn. 25.5.2.1), die in den allermeisten Fällen ohne „Dilemma“ zu einer Verbesserung gegenüber der heutigen Ausgangssituation führt: Der naturwissenschaftliche Vergleich des maschinellen Entscheidungsdilemmas mit dem Fahrer heute wird voraussichtlich nämlich ergeben, dass diesem in der ansonsten gleichen Situation - schon aufgrund der zusätzlich zuzugestehenden Schreckzeit (vgl. [14]) - heute kein Schuldvorwurf gemacht wird. Auch kommt es in „Dilemma-Situationen“ unterschiedslos im Fall eines menschlichen Fahrers wie im Fall maschinellen Wirkens letztlich zu einer Schädigung. Der Grund für die Befassung mit der Frage wäre deshalb auf die grundsätzlich erfreuliche Entwicklung zurückzuführen, dass derartige Situationen aufgrund technischer Entwicklung beeinflussbar geworden wären und so regelmäßig die Rettung konkret gefährdeter Rechtsgüter ermöglicht wird. Dem Gewicht dieses Arguments wird man sich voraussichtlich nicht verschließen können. 


\subsubsection{Möglichkeit zur Übersteuerung durch die Passagiere}

Die Frage der Übersteuerungsmöglichkeit von Fahrzeugen resultiert aus der in der Vergangenheit geführten Diskussion zu Fahrerassistenzsystemen, die im Wesentlichen ihren Ausgangspunkt in den nach aktuellem Stand (August 2014) noch gültigen konkreten Formulierungen des Wiener Übereinkommens über den Straßenverkehr von 1968 nimmt [17]. Auch wenn dies nicht konkret angesprochen wurde, ist dieser Grundgedanke zugleich verwandt mit der Frage nach den Pflichten des Fahrers bei vollautomatisierter Fahrzeugführung, die in Bezug auf das geltende Verhaltensrecht in Deutschland als widersprüchlich beschrieben worden ist [10]. Der Zusammenhang lässt sich anhand des Begriffes der „Dominanz“ bzw. „Autorität“ beschreiben, der im Rahmen des vorliegenden Projektes den „Use-Cases“ (s. Kap. 2) zugrunde gelegt wurde: Alle vier stellvertretenden Applikationen sehen einen Einfluss des Fahrers bzw. Passagiers auf die Fahrzeugsteuerung vor, der mit zunehmender Reichweite der Autonomie immer weiter zurücktritt, da anderen Instanzen im Rahmen der Funktionsumsetzung Vorrang einzuräumen ist. Bereits die vorliegend betrachtete Stellvertreterapplikation mit der geringsten Autonomie, der „Autobahnautomat mit Verfügbarkeitsfahrer - Autobahnpilot“ sieht aber vor, dass der Fahrer „... während der autonomen Fahrt zum einfachen Passagier ..." wird, sodass er ,... einer anderen Tätigkeit nachgehen [kann]“. Hierdurch fehlt es bereits vollständig an einer Grundlage für die jederzeitige Übersteuerungsmöglichkeit, die von vornherein immer faktisch (und nicht nur technisch) für notwendig erachtet worden ist. Die faktische Übersteuerungsmöglichkeit des Fahrers war eine Umschreibung für die bei Fahrerassistenzsystemen seinerzeit noch nicht infrage gestellte „Letztentscheidungsbefugnis des Fahrers“ und damit die untergeordnete Rolle von Fahrerassistenzsystemen gegenüber dem Fahrer bei der Fahrzeugsteuerung, die nur eine abgeleitete Handlungs- und Entscheidungsbefugnis des Systems für möglich hielt (s. Abschn. 25.4.1). Autonome Fahrzeuge stellen diese Situation gänzlich infrage, sodass es nicht sinnvoll erscheint, aus Vorschriften, die einen menschlichen Fahrer bei der Fahrzeugsteuerung zugrunde legen, Ableitungen zu treffen, die Wirkung für die Zukunft entfalten. Dieser grundlegende Wandel zum eigenständigen maschinellen Wirken ist bereits in Abschn. 25.4.2 beschrieben.

Mit Blick auf die Grundrechte lässt sich in diesem Zusammenhang allerdings in aller gebotenen Kürze auf einen Aspekt hinweisen: Die in Art. 2 GG normierten Freiheitsrechte von Passagieren autonomer Fahrzeuge stellen den großen Rahmen dar, der - soweit vorliegend von Relevanz - sich insbesondere auf die Freiheit zur körperlichen Fortbewegung und die freie Entfaltung der Persönlichkeit bezieht (vgl. [3]). Die sich hieraus ergebenden Einschränkungen der Freiheitsausübung scheinen nach aktuellem Stand der Diskussion jedoch nicht grundlegend infrage gestellt, solange den Passagieren autonomer Fahrzeuge stets die Möglichkeit zur Verfügung steht, ein Anhalten des Fahrzeuges an der nächsten sicheren und geeigneten Stelle zu veranlassen. 


\subsubsection{Fehlerkompensationsfähigkeit beim autonomen Fahren}

Eine ,grundlegende Annahme“ des vorliegenden Projektes liegt darin, ,,dass die Use-Cases im Betrachtungszeitraum in einem Mischbetrieb aus Verkehrsmitteln mit unterschiedlichen Automatisierungsgraden ... von ,driver only“ über ,,assistiert“ bis ,,vollautomatisiert“ ... eingesetzt werden“" (s. Kap. 2). Insoweit ist zu fordern, dass sich autonome Fahrzeuge in das bestehende Verkehrssystem einfügen.

Im Fall herkömmlicher Fahrzeugführung ist, wie auch im Fall der Verwendung heute verfügbarer Fahrerassistenzsysteme (im weiteren Sinn), denen niemals eine eigenständige Handlungsqualität zukommt (s. Abschn. 25.4.1), die Straßenverkehrsordnung uneingeschränkt anwendbar. Der Fahrer bewegt sich demnach heute im Spannungsfeld zwischen „Vertrauensgrundsatz“ und „defensiver Fahrweise“: Der „Vertrauensgrundsatz“ besagt, dass der sich selbst verkehrsrichtig verhaltende Verkehrsteilnehmer unter normalen Verhältnissen nicht vorsorglich alle möglichen (seltenen) Verkehrsverstöße anderer Verkehrsteilnehmer in Erwägung ziehen muss. Der „Vertrauensgrundsatz“ ist notwendig, um den Verkehrsfluss aufrecht zu erhalten. Zugleich soll der Fahrer heute „defensiv fahren“ (um der Verkehrssicherheit Rechnung zu tragen) und somit mehr als die gebotene Sorgfalt anwenden (was einen teilweisen Verzicht auf Vertrauen in verkehrsrichtiges Verhalten anderer bedeutet), ohne dass dies jedoch den Vertrauensgrundsatz ,, grundsätzlich “ infrage stellt [14]. Wie diese Anforderungen im Einzelfall, also in Bezug auf den Fahrer in bestimmten Verkehrssituationen verstanden werden, ließe sich in Deutschland aus Urteilen ableiten, wobei die kontinental-europäische Ausrichtung auf systematisiertes Gesetzesrecht allenfalls eine Orientierung in dieser Hinsicht erlaubt, aber kein Präjudiz für den konkreten Einzelfall bedeutet, sodass der Wert einer solchen Zusammenstellung als eher gering einzuschätzen ist.

Von autonomen Fahrzeugen, die sich im „Mischbetrieb“ bewegen, wird im Ergebnis zu fordern sein, dass sie (mindestens) denselben Anforderungen genügen, die auch der Fahrer erfüllen soll - schon um hierdurch keine neuen Gefahren zu verursachen. Bei der präzisen Beschreibung der Anforderungen an eine Fehlerkompensationsfähigkeit ist jedoch festzustellen, dass eine erhebliche Unschärfe verbleibt. Überträgt man deshalb die heutigen rechtlichen Anforderungen im Straßenverkehr in eine Leistungsdefinition einer autonomen Steuerungsfunktion, lässt sich mit ausreichender Sicherheit nur festhalten, dass Fehlerkompensationsfähigkeiten autonomer Fahrzeuge grundsätzlich erforderlich sind: Autonome Fahrzeuge müssten eindeutig erkennbares Fehlverhalten anderer Verkehrsteilnehmer im Rahmen der Fahrzeugsteuerung berücksichtigen und die maschinelle Steuerung hieran anpassen. Wie dies konkret zu geschehen hat, wird nach heutigem Recht im Einzelfall der Einschätzung des Fahrers überlassen. Allein diese Anforderung technisch umzusetzen, würde eine hohe Leistungsfähigkeit der Umfelderkennung und ausgesprochen komplexe Abwägungsvorgänge voraussetzen; sie werden allerdings - auch im Fall des Fahrers - nicht näher definiert, sondern grundsätzlich im Rahmen der geforderten Fahreignung und Fahrbefähigung von Fahrern vorausgesetzt [18]. Die fehlende Konkretisierung der Anforderungen kann sich deshalb als zu anspruchsvoll für die Umsetzung als maschinelle Steuerung erweisen. 
Angesichts resultierender Unsicherheit durch die antagonistische Forderung ,defensiven Fahrens" bleibt somit vorerst nur die - den Verkehrsfluss möglicherweise infrage stellende - Übererfüllung von Fehlerkompensationsfähigkeit im Sinne eines Risikoausschlusses. Dabei bleibt allerdings die Frage offen, ob tatsächlich mit einer erheblichen Einschränkung des Verkehrsflusses zu rechnen ist: Durch die oben dargelegte Erweiterung der Einflussmöglichkeit maschineller Steuerung (s. Abschn. 25.5.2) könnten sich maschinelle Fahrzeugsteuerungen gegenüber dem Fahrer als deutlich leistungsfähiger erweisen. Dieser Vorteil, der derzeit nicht zu bemessen ist, könnte Nachteile für den Verkehrsfluss ausgleichen (wobei der resultierende Umfang ebenso offen bleibt).

In der Frage von Fehlerkompensationsfähigkeiten autonomer maschineller Steuerungssysteme könnte es sich zur Verbesserung der Rechtssicherheit als sinnvoll erweisen, einheitliche, hinsichtlich des Zieles definierte Anforderungen (vergleichbar mit Wirkvorschriften etwa im Bereich von Fahrzeugbremsen) zu formulieren, die den Stand von Wissenschaft und Technik niederlegen und harmonisieren. Ein solcher Leistungskatalog dürfte bei der juristischen Bestimmung von realistischen Leistungsanforderungen an autonome Steuerungssysteme Wirkung entfalten.

\subsubsection{Kommunikation im Straßenverkehr}

Hinsichtlich der Kommunikation ist für den Straßenverkehr von den heute bestehenden Möglichkeiten auszugehen. Der Gebrauch einiger fahrzeugseitiger Licht- und Schallzeichen - wie die (Licht-)Hupe, der Fahrtrichtungsanzeiger oder die Warnblinkanlage - wird von der StVO, soweit vorhanden, ausdrücklich vorausgesetzt. So sind beispielsweise Warnzeichen (Gebrauch des Warnblinklichts) im Fall von Gefahren bei Schulbussen, wenn Fahrgäste einoder aussteigen oder als Warnung im Fall der Annäherung an ein Stauende, dem Liegenbleiben defekter Fahrzeuge und dem Abschleppen vorgesehen (vgl. $\S \S 15,15 \mathrm{a}, 16$ Straßenverkehrsordnung). Die Verwendung von Fahrtrichtungsanzeiger und (Licht-)Hupe ist durch $\S 5$ Abs. 4a und 5 Straßenverkehrsordnung im Rahmen eines Überholvorgangs angeordnet bzw. zugelassen. Der Gebrauch des Fahrtrichtungsanzeigers beim Abbiegen (§9 Abs. 1 Straßenverkehrsordnung) oder Anfahren (§ 10 Straßenverkehrsordnung) ist, soweit vorhanden, vorgeschrieben. Über diese normativ formalisierte Kommunikation erreicht die Straßenverkehrsordnung eine Erwartungserleichterung bei anderen Verkehrsteilnehmern [19].

Daneben hat sich eine informelle (oder rechtlich nicht abgesicherte) Verwendung von (Licht-)Zeichen eingebürgert, die allerdings nicht durch die Straßenverkehrsordnung vorgesehen ist (so kann in Deutschland die Lichthupe auch als Vorfahrtsverzicht aufzufassen sein). Hierbei ist allerdings anzumerken, dass eine solche Zeichenverwendung bereits nicht mehr dem Schutz des Vertrauensgrundsatzes im Straßenverkehr unterliegt, weil sie nicht als „,verkehrsrichtiges“ Verhalten eingeordnet werden kann (und im Übrigen einer erheblichen Gefahr der Fehlinterpretation unterliegt).

Die unmittelbare verbale Kommunikation aus geschlossenen Fahrzeugen im Straßenverkehr wird erschwert, wenn nicht unmöglich gemacht durch die physische Trennung des 
Fahrers von der Umwelt und den Lärmpegel der Umgebung. Auch die informelle Verständigung über einfache Gesten ist aufgrund von Spiegelungseffekten bei den heute üblichen getönten und geneigten Fahrzeugscheiben deutlich eingeschränkt. Dennoch lässt sich auch der Straßenverkehrsordnung im Fall „,besonderer Verkehrslagen“ eine Anwendungsbestimmung entnehmen, die nicht näher bestimmte informelle Kommunikation vorsieht (als spezialgesetzliche Ausprägung des Vorsichts- und Rücksichtnahmegebotes des $\S 1$ Abs. 1 Straßenverkehrsordnung): So ist bei erforderlichem Vorfahrtsverzicht eine Verständigung mit dem Verzichtenden im Rahmen von $\S 11$ Abs. 3 Straßenverkehrsordnung erforderlich, die allerdings voraussetzt, dass diese Verständigung eindeutig erfolgt hier kann sogar im Ausnahmefall die Lichthupe unterstützend wirken [14].

Angesichts bestehender Herausforderungen bei der Kommunikation (s. auch Kap. 7) kann auch ein vorwegnehmendes Handeln, insbesondere dann, wenn es sich in Bewegung ausdrückt, ein Zeichen für die intendierten Zielhandlungen sein [19], sodass auch dem von außen wahrnehmbaren Verhalten im Straßenverkehr wie dem Anhalten, Abbremsen und Anfahren erhebliche Bedeutung im Rahmen der informellen Kommunikation zukommt. Ausdrücklich vorgesehen wird solches Verhalten beispielsweise auch durch $\S 8$ Abs. 2 StVO, wonach dem im Rahmen einer Vorfahrtsregelung Wartepflichtigen aufgegeben wird, durch sein „,... Fahrverhalten ... erkennen [zu] lassen, dass gewartet wird.“

Neben der Nonverbalität und Anonymität wird insbesondere auch die Komplexität der Situation als charakteristische Randbedingung für die Kommunikation im Straßenverkehr beschrieben. Diese Komplexität ist insbesondere durch die Schnelligkeit und Flüchtigkeit der Kommunikation im Straßenverkehr bestimmt [20].

Daraus lassen sich erste wichtige Schlussfolgerungen für das autonome Fahren ableiten: Während die normierte, formelle Kommunikation sich möglicherweise nur als eine Herausforderung für die maschinelle Wahrnehmung und Interpretation darstellt und sich eventuell in der umgekehrten Richtung noch maschinell programmieren lässt, ist hier bereits als Frage aufzuwerfen, inwieweit für andere Fahrer überhaupt Erkennbarkeit maschineller Steuerung gegeben ist oder sich umsetzen ließe. Als Folge stellt sich im ,gemischten“ Kommunikationsverhältnis mit Maschinen die Frage, ob andere Fahrer auf den Inhalt erfolgter formeller Kommunikation vertrauen. Das setzt mindestens Kenntnis von diesbezüglichen Fähigkeiten autonomer Fahrzeuge voraus, damit kommunikative Interaktion funktioniert. Gegebenenfalls ergibt sich sogar ein Bedürfnis anderer Verkehrsteilnehmer, die Inhalte maschineller Kommunikation auch im Nachhinein (beispielsweise nach einem Unfall) noch nachvollziehen zu können, um die Bereitschaft aufzubringen, auf maschinelle Kommunikationsinhalte zu vertrauen.

Während die sich ergebenden Herausforderungen im Fall der formellen Kommunikation noch lösbar erscheinen, ist informelle Kommunikation im Mischverkehr von nochmals größeren Herausforderungen geprägt (Mischverkehr liegt der vorliegenden Betrachtung insgesamt zugrunde (s. Kap. 2). Es ergibt sich in diesem Bereich ein Bedürfnis, zu Lösungsansätzen zu kommen, die eine kommunikative Brücke zwischen maschinell gesteuerten Fahrzeugen und den übrigen Verkehrsteilnehmern vermitteln. Letztlich liegt diesem Konflikt die bereits in Abschn. 25.5 beschriebene Ursache zugrunde, dass eine ins Einzelne 
gehende Regelung von Kommunikationsverhalten bei menschlichen Fahrern im Straßenverkehr nicht zwingend erforderlich ist und die Umsetzung den Verkehrsteilnehmern im Einzelnen überlassen bleiben kann. Diese informelle Kommunikation zwischen Verkehrsteilnehmern mag zwar konfliktträchtig und verbesserungsbedürftig sein [20], dennoch ist anzunehmen, dass Fahrer regelmäßig zur Lösung dieser Herausforderung in allen Situationen in der Lage sind - zumal dies erst die im Straßenverkehr erforderliche situationsindividuelle Anpassung garantiert. Bei Einführung einer maschinellen Fahrzeugsteuerung fehlt den Maschinen diese den Menschen eigene Fähigkeit, eine nicht näher spezifizierte Verständigung herbeizuführen, gegebenenfalls fehlt es bereits an der maschinellen Wahrnehmungsfähigkeit, die Notwendigkeit von Kommunikation überhaupt zu erkennen: Voraussetzung menschlicher Kommunikation ist das Herstellen einer reflexiven Aufmerksamkeit der Kommunikationspartner und Wahrnehmung untereinander [19]. Hier zeigt sich deutlich, dass die „gemischte“ Kommunikation noch grundlegender Ansätze zur Überbrückung dieser kommunikativen Kluft mit Maschinen erfordert. Auch dürfte dies eine Grundvoraussetzung für das Funktionieren eines gemischt fahrerisch und maschinell gesteuerten Straßenverkehrs sein.

Gleichwohl lässt sich für die Realisierung autonomen Fahrens die Bedeutung von Kommunikation danach abstufen, in welchen Situationen vor allem die für eine technische Umsetzung sehr herausfordernd erscheinende informelle Kommunikation von Gewicht ist: Die oben beschriebene Schnelligkeit und Flüchtigkeit der Kommunikation im Straßenverkehr dürfte sich wesentlich nach Umgebungsbedingungen und Fahrgeschwindigkeiten unterscheiden. Einfach strukturierte, extrem flüchtige Umgebungsbedingungen wie auf einer Autobahn, die schon aufgrund der gefahrenen Geschwindigkeiten die weitgehende Verwendung formeller Kommunikation erfordern, könnten eine Umsetzung autonomen Fahrens dort wesentlich erleichtern und ein Anpassungsbedürfnis autonomer Fahrzeuge an den Bedarf nach informeller Kommunikation weniger dringlich erscheinen lassen (als beispielsweise eine innerörtliche Verkehrsumgebung).

Auch ist anzunehmen, dass die Herausforderung, die in der Kommunikation liegt, nur im gemischten Verkehr auftritt, während sie insbesondere zwischen autonomen Maschinen technisch gut lösbar erscheint (über kooperative Systeme). Für den gemischten Verkehr ist zudem zu untersuchen, welche Bedeutung dem vorwegnehmenden Handeln zukommt, das sich in Bewegung ausdrückt, und wie es für eine künftige Kommunikation im Straßenverkehr genutzt werden kann. Insbesondere ist von Bedeutung, welche Situationen möglicherweise hierdurch eindeutig gelöst werden könnten. Hierbei ist aber zugleich die Gefahr zu berücksichtigen, wonach ein maschinelles Fahrsystem - durch eine stark die Fehlerkompensationsfähigkeiten anderer Verkehrsteilnehmer berücksichtigende Auslegung (s. Abschn. 25.5.4) möglicherweise die Gefahr birgt, Einbußen für den Verkehrsfluss zu bedeuten, wenn diese durch andere Verkehrsteilnehmer zum eigenen Vorteil ausgenutzt werden.

Abschließend ist hinsichtlich der Kommunikation im Straßenverkehr festzuhalten, dass auch hier ein Katalog der technisch umsetzbaren Kommunikationsstrategien für autonome Fahrzeuge im Sinne eines Leistungskataloges ratsam erscheint, sobald dies technisch absehbar wird. Diese Zusammenstellung von Leistungsmerkmalen autonomer Fahrzeuge im 
Hinblick auf die Kommunikation wird insbesondere im gemischten Verkehr langfristig erforderlich sein und könnte die Diskussion um mögliche Kommunikationskonzepte wesentlich voranbringen.

\subsubsection{Regelübertretung}

Über $\S 24$ Straßenverkehrsgesetz als Blankettnorm in Verbindung mit den Ordnungswidrigkeitstatbeständen aus der Straßenverkehrsordnung ( $\$ 49)$, Fahrzeugzulassungsverordnung (§ 48), Straßenverkehrszulassungsordnung ( 6 69a) und Fahrerlaubnisverordnung (§ 75) werden Verstöße gegen Verbote, Gebote und Anordnungen an Verkehrsteilnehmer und Fahrzeughalter im Straßenverkehr im Wesentlichen geahndet [14]. Als Straftat gelten darüber hinaus Delikte im Zusammenhang mit dem Straßenverkehr aus dem 28. Abschnitt des Strafgesetzbuches (StGB) zu gemeingefährlichen Straftaten (im vorliegenden Zusammenhang sind insbesondere Eingriffe von außen in den Straßenverkehr gemäß $§ 315 b$ StGB und Gefährdungen im Straßenverkehr gemäß § 315c StGB von einiger Bedeutung). Es liegt auf der Hand, dass ein maschinelles Steuerungsverhalten autonomer Fahrzeuge die Anwendung dieser Vorschriften ausschließt: Es wird bei Ordnungswidrigkeiten und Strafvorschriften stets an ein menschliches Handeln angeknüpft, an dem es vorliegend mangelt.

Die bestehenden Vorschriften, die strafrechtlich und als Ordnungswidrigkeit sanktioniert sind, haben gleichwohl eine Indizwirkung dahingehend, welches Verhalten im Straßenverkehr nicht nur die öffentliche Ordnung, sondern, insoweit hier von besonderem Interesse, gerade auch die öffentliche Sicherheit besonders infrage stellen kann. Die Verkehrsregeln sind dabei gleichwohl ,... elastisch (verkehrsgerecht) und ohne Kleinlichkeit zu handhaben und auszulegen ...", dies gilt auch, soweit es sich um Spezialregelungen handelt, die gegebenenfalls behindern oder gefährden können [14]. Darüber hinaus wird durch den in $\S 16$ des Gesetzes über Ordnungswidrigkeiten geregelten Tatbestand des rechtfertigenden Notstandes ein gesetzlicher Rechtfertigungsgrund normiert, der bei gegenwärtigen, nicht anders abwendbaren Gefahrenlagen für Leben, Leib, Eigentum oder anderen Rechtsgütern erlaubt, auch Verkehrsregeln zuwider zu handeln, um die drohende Gefahr abzuwenden. Allerdings ist ein wesentliches Überwiegen der Gefahren erforderlich und die Angemessenheit der Gefahrenabwehr zu berücksichtigen. Wenngleich die entsprechenden Fälle restriktiv gehandhabt werden müssen, schon um Schutzbehauptungen zu vermeiden, handelt es sich hierbei letztlich um ein normatives Instrument, um eine interessengerechte Abwägung verschiedener grundrechtlich geschützter Rechtsgüter im Einzelfall widerspruchsfrei - und damit verfassungskonform - zu ermöglichen. Die Regelübertretung selbst unterliegt demselben Maßstab, sodass sie ihre äußerste Grenze für den Straßenverkehr letztlich in der „Gefahrenschwelle“ für andere Verkehrsteilnehmer findet (vgl. [21] zur Frage der Berücksichtigung von Verkehrssicherheit im Rahmen der Abwägung im Tatbestand des rechtfertigenden Notstands gemäß $§ 16$ Gesetz über Ordnungswidrigkeiten). 
Wird vor diesem Hintergrund die Einführung autonomen Fahrens berücksichtigt, ist anzunehmen, dass die Interessenlage sich nicht verändert - jedenfalls für den Mischverkehr von autonomen und fahrergesteuerten Fahrzeugen, der hier zugrunde gelegt wird (s. Kap. 2). Eine Änderung könnte dann eintreten, wenn - aufgrund einer sehr weitgehenden maschinellen Beherrschung des mit der Regelübertretung einhergehenden maschinellen Steuerungsrisikos - die beschriebene Abwägung ergibt, dass eine Risikoerhöhung durch die Regelübertretung nicht eintritt. Damit wäre dann aber zugleich die Sinnhaftigkeit der betroffenen Regel als solche infrage gestellt: Die „Übertretung“ der Regel würde sich dann im Zusammenhang mit dem Mischverkehr nicht nachteilig auswirken.

Denkbar ist allerdings auch, dass autonomes Fahren dazu führt, dass Verkehrsregeln wesentlich detaillierter erforderlich wären und wesentlich weniger flexibel gehandhabt werden könnten (was wiederum die Frage gesellschaftlicher Akzeptanz aufwerfen würde). Für eine solche Einschränkung der bestehenden Situation wäre wiederum zunächst erforderlich, die technische Steuerungsleistung autonomer Fahrzeuge in einem Leistungskatalog näher zu beschreiben, sobald sie technisch absehbar wird. Dies würde erlauben, die Konsequenzen auch im Fall von Regelübertretungen wesentlich grundlegender zu erörtern, als dies nach gegenwärtigem Stand noch möglich ist.

\subsection{Spezielle Rechtsfragen autonomen Fahrens}

Hinsichtlich der ordnungsrechtlichen, produkt- und straßenverkehrshaftungsrechtlichen Bewertung vollautomatisierten, fahrerlosen Fahrens kann auf die bereits einleitend gemachten Ausführungen verwiesen werden (s. Abschn. 25.4.2 sowie die Einleitung zu Abschn. 25.5). Zusammenfassend lässt sich hierzu vor allem feststellen, dass insbesondere die ordnungsrechtlichen und straßenverkehrshaftungsrechtlichen Vorschriften (hier vor allem das Straßenverkehrsgesetz, die Straßenverkehrsordnung, Fahrerlaubnisverordnung, Straßenverkehrszulassungsordnung) zum Zeitpunkt ihrer Entstehung nur berücksichtigen konnten, was als Stand der Technik im Straßenverkehr bekannt war und als Regelungsgegenstand einbezogen wurde: Es wird durchgängig im öffentlichen Straßenverkehr von einem Fahrer ausgegangen, der die Fahrzeugsteuerung selbst vornimmt. Dabei ist auch hinsichtlich aller heute marktverfügbaren Systeme zu beobachten, dass sie gerade keine eigenständige Entscheidungsqualität (s. Abschn. 25.4.1) besitzen, sondern voraussetzen, dass der Fahrer mindestens ,als Überwacher" aktiv ist.

\subsubsection{Ordnungsrechtliche Bewertung fahrerloser Fahrzeuge}

Wird die ordnungsrechtliche Bewertung vollautomatisierten Fahrens [10] deshalb nochmals erweitert auf gänzlich unbemannte Fahrzeuge und Insassen, die nicht in der Lage sind, das Fahrzeug selbst zu führen, ist letztlich festzustellen, dass sich an der bereits bestehen- 
den Unvereinbarkeit mit heutigem Ordnungsrecht, das diese technologische Veränderung bislang noch nicht berücksichtigt, zunächst einmal nichts ändert.

Gleichwohl ist darauf hinzuweisen, dass sich fahrerlose oder vollständig autonome Fahrzeuge (s. Use-Cases: „Autonomes Valet-Parken“ und „Vehicle-on-Demand“, Kap. 2) in den sich ergebenden Anforderungen an den rechtlichen Rahmen deutlich von solchen unterscheiden, die einen „Verfügbarkeitsfahrer“ voraussetzen: Mit der fortbestehenden Möglichkeit einer Fahrzeugführung durch den Fahrer sind für bestimmte Fahrtabschnitte oder im Fall des Übernahmewunsches durch den Fahrer dieselben Anforderungen grundlegender Art (Fahreignung und Fahrbefähigung, vgl. hierzu [18]) zu erfüllen, die bereits heute an einen Fahrer gestellt werden - im Fall des ,,Vollautomaten mit Verfügbarkeitsfahrer" jedenfalls dann, wenn der Fahrer von der Möglichkeit, als Fahrer selbst zu steuern (also eigenständige Fahrqualität auszuüben), Gebrauch zu machen intendiert. Insoweit bedarf es keines grundlegend anderen, sondern eines um notwendige Vorschriften für autonome Fahrzeuge und damit eigenständige maschinelle Entscheidungsqualität ergänzten Rechtsrahmens. Mit Blick auf diese eigenständige maschinelle Entscheidungsqualität kommt man deshalb für alle Use-Cases in rechtlicher Hinsicht zu demselben Ergebnis. Der Wandel liegt vor allem in der Ermöglichung des eigenständigen maschinellen Wirkens und seiner Ausgestaltung. Die Ausgestaltung maschineller Fahrzeugsteuerung in ordnungsrechtlicher Hinsicht ist heute ungeregelt, obwohl eine Regelung - zumindest in sinngemäßer Anwendung mindestens der verhaltensrechtlichen Vorschriften der Straßenverkehrsordnung als technische Wirkvorschriften - angezeigt erscheint.

Sofern sich ein Regelungsbedürfnis autonomen Fahrens daher nicht bereits aus dem neuartigen maschinellen Automatisierungsrisiko selbst ergibt (s. Abschn. 25.5.1), erscheint zumindest im Hinblick auf den Einsatz entsprechender Funktionen im gemischten Verkehr hinsichtlich der Fehlerkompensationsfähigkeiten (s. Abschn. 25.5.4) und des Kommunikationsverhaltens (s. Abschn. 25.5.5) autonomer Fahrzeuge eine ordnungsrechtliche Regelung aber angezeigt. Bei der ordnungsrechtlichen Regelung solchen Interaktionsverhaltens wird zudem sehr genau zu berücksichtigen sein, wie weit die Rolle des Fahrers im Einzelfall überhaupt reicht: Berücksichtigt man die sich ergebenden technischen Möglichkeiten für eine im Einzelfall auch allein maschinell geprägte Mobilität (wie in den Use-Cases „Vollautomat mit Verfügbarkeitsfahrer“ oder ,Vehicle-on-Demand“ beschrieben), erscheinen die bislang grundlegenden Anforderungen an Fahreignung und Fahrkompetenz bereits verzichtbar. Durch die vielfältigen Möglichkeiten könnte es deshalb letztlich zu einer bislang nicht gekannten Vielfalt ordnungsrechtlicher Vorgaben für die Teilnahme am Straßenverkehr mit (autonomen) Fahrzeugen kommen, die sich im Wesentlichen danach unterscheiden könnte, wie weit maschinelle Steuerungsqualität reicht und in welchem Umfang sie genutzt werden soll. 


\subsubsection{Bewertung autonomen Fahrens nach dem Haftungsrecht im Straßenverkehr}

Hinsichtlich der mit dem autonomen Fahren einhergehenden Veränderung zu einem eigenständigen maschinellen Wirken bei der Fahrzeugsteuerung ist auch für die Bewertung des Haftungsrechtes im Straßenverkehr grundlegend festzustellen, dass die bestehenden Vorschriften diese Veränderung nicht berücksichtigen konnten.

\subsubsection{Halterhaftung}

Gleichwohl besteht in diesem Bereich eine grundlegende Haftungsvorschrift für Kraftfahrzeuge ( $\$ 7$ Straßenverkehrsgesetz), wonach der Halter des Fahrzeuges verschuldensunabhängig für den Fahrzeugbetrieb zum Ersatz aller kausal hierauf zurückzuführenden Schäden, die nicht Vermögensschäden sind, verpflichtet wird (einziger verbleibender Haftungsausschlussgrund ist die höhere Gewalt, § 7 Abs. 2 Straßenverkehrsgesetz). Diese Beschränkung auf den Fahrzeugbetrieb als haftungsauslösendes gesetzliches Tatbestandsmerkmal unterscheidet deshalb bereits heute nicht danach, ob es sich um einen Schaden handelt, der durch fahrerisches Steuerungsverhalten oder durch technisches Versagen verursacht wird. Da auch maschinelle Steuerungsentscheidungen, die kausal einen Schaden verursachen, sich widerspruchsfrei als technisches Versagen einordnen lassen, treten in dieser Hinsicht keine grundlegenden Widersprüche auf.

Fahrzeughalter ist, wer das Fahrzeug für eigene Rechnung gebraucht, also insbesondere die Nutzungen zieht und die Kosten bestreitet und somit auch berechtigt ist, über die Benutzung des Fahrzeuges als Gefahrenquelle zu entscheiden [14]. Geht man davon aus, dass auch fahrerlosen Fahrzeugen ein Halter zuzuordnen ist, was widerspruchsfrei nach geltendem Recht möglich wäre, würde in dieser Hinsicht auch bei solchen Fahrzeugen keine Unvereinbarkeit mit geltendem Recht auftreten. Autonomes Fahren würde dennoch den Gegenstand der Halterverantwortung verändern - allerdings ist die Halterverantwortung für autonomes Fahren nach dem heutigen Wortlaut der Vorschrift bereits mit umfasst: Die bislang durch den Fahrer erfolgende Fahrzeugsteuerung wird durch eine maschinelle Fahrzeugsteuerung ersetzt (soweit der Anwendungsbereich der jeweiligen autonomen Steuerung reicht). Für die Halterverantwortung ergeben sich daher nicht neue Pflichten, sondern eine völlig andere, neue Steuerungsqualität, die zivilrechtliche Haftung auslöst.

\subsubsection{Fahrerhaftung und Unfalldatenaufzeichnung}

Andere für die zivilrechtliche Haftung relevante Vorschriften des Straßenverkehrsgesetzes legen hingegen eindeutig die Steuerung eines Fahrers zugrunde, so beispielsweise $\S 18$ Abs. 1 Straßenverkehrsgesetz, wonach in den Fällen der Halterhaftung zugleich eine Haftung des Führers des Kraftfahrzeuges vermutet wird (vgl. Satz 2). Damit liegt aber auch $\S 18$ Straßenverkehrsgesetz letztlich nur wieder die bislang uneingeschränkt korrekte Annahme zugrunde, wonach stets ein Fahrer für das Steuerungsverhalten des Fahrzeuges zuständig ist. Letztlich unterscheidet sich deshalb die haftungsrechtliche Bewertung von fahrerlosen Fahrzeugen inhaltlich nicht von vollautomatisierten [10] Fahrzeugen. Entspre- 
chend verhält es sich auch mit den bislang für deliktisches Verhalten von Fahrern anwendbaren Vorschriften des Bürgerlichen Gesetzbuches (beispielsweise $§ 823$ Abs. 1 BGB oder $\S 823$ Abs. 2 BGB in Verbindung mit verletzten Vorschriften der StVO), die gleichermaßen bei eigenständiger maschineller Fahrzeugsteuerung diese nicht mehr erfassen können (da sie an ein menschliches Handeln anknüpfen, das bei autonomen Fahrzeugen nicht gegeben ist).

Bei den vorliegend betrachteten autonomen Fahrzeugen, die zum Teil nicht einmal die Anwesenheit oder gelegentliche Steuerung durch einen Fahrer bedingen, ist infrage zu stellen, inwieweit für den Regelfall noch von der Fähigkeit der Fahrzeuginsassen auszugehen ist, einen Unfallhergang beschreiben zu können. Damit wird deutlich, dass bei der Frage des inneren Ausgleichs von Unfallschäden, die unter Beteiligung von zwei oder mehreren autonomen Fahrzeugen auftreten, die Schadensaufteilung nach geltendem Recht und damit $\S 17$ Straßenverkehrsgesetz vor dem Problem steht, dass das vorwiegend zur Anwendung kommende „Maß der Verursachung“ [14] nicht mehr - wie heute aber üblich - mindestens auf eine Vernehmung der Fahrer (zumeist zugleich zivilrechtliche Prozesspartei, gegebenenfalls aber auch Zeuge) gestützt werden kann: Dem liegt vor allem die Rollenveränderung zugrunde, wonach der Fahrer ,zum einfachen Passagier“ (s. Kap. 2) wird und deshalb zum Unfallhergang eher ausnahmsweise und nur aufgrund zufälliger Beobachtung Angaben wird machen können. Insoweit tritt eine Veränderung ein, die bereits für das Haftungsrecht im Straßenverkehr erforderlich erscheinen lässt, dass technische Vorkehrungen (wie eine Unfalldatenaufzeichnung während automatisierter Steuerung) als Grundlage für eine entsprechende Schadensaufteilung getroffen werden, sofern man haftungsrechtlich an diesem Aufteilungsprinzip nach Verursachungsanteilen auch für das autonome Fahren festhalten möchte (was durchaus möglich erscheint).

\subsubsection{Bewertung nach dem Produkthaftungsrecht}

Der Bereich der Produkthaftung für die automatisierte Steuerung autonomer Fahrzeuge wurde bereits in Abschn. 25.5.1 behandelt. Demnach kommt der Frage nach der im Einzelfall relevanten Ursache für einen Unfallschaden im Sinne naturwissenschaftlicher Kausalität entscheidende Bedeutung zu.

\subsubsection{Produkthaftungsrechtliche Bedeutung eines Unfalldatenschreibers für das autonome Fahren}

Darüber hinaus ergibt sich aber für die Fälle autonomen Fahrens, die einen „Verfügbarkeitsfahrer“" voraussetzen (s. Use-Cases „Autobahnautomat mit Verfügbarkeitsfahrer Autobahnpilot“ und „Vollautomat mit Verfügbarkeitsfahrer“, Kap. 2), noch ein weiterer Aspekt, der wiederum im Zusammenhang mit der Frage eines Unfalldatenschreibers steht: Sowohl im Hinblick auf das Produkthaftungsrecht (als auch ordnungsrechtlich hinsichtlich bußgeldbewehrter Verstöße gegen das Ordnungsrecht) liegt es nahe anzunehmen, dass als Schutzbehauptung durch einen aktiven Verfügbarkeitsfahrer vorgebracht werden könnte, 
nicht selbst, sondern mithilfe des autonomen Steuerungssystems das Fahrzeug geführt zu haben. Will man entsprechende Schutzbehauptungen ausschließen, wird man sowohl zur produkthaftungsrechtlichen Absicherung des Herstellerinteresses als auch zur Erbringung des Nachweises im Rahmen des Ordnungswidrigkeitenrechtes kaum umhin kommen, eine Aufzeichnung von Daten bei der Fahrzeugsteuerung - mindestens während autonomer Steuerungsdauer - in Erwägung zu ziehen. Die Möglichkeit hierzu erscheint unter dem Aspekt des Datenschutzes zunächst nicht ausgeschlossen, sofern die Daten im Fahrzeug verbleiben, das Transparenzgebot gewahrt wird und die Datenverarbeitung nur im erforderlichen Umfang erfolgt, Daten mithin beispielsweise nur im Fall eines Unfallschadens dauerhaft gespeichert würden. Darüber hinaus ist eine Einschränkung der Datenaufzeichnung auf die Zeitdauer maschineller Steuerung in Erwägung zu ziehen, was dem Gebot der Datensparsamkeit (§ 3a Bundesdatenschutzgesetz), das sich im Übrigen gleichermaßen aus dem Verhältnismäßigkeitsprinzip jeder Datenverarbeitung ergeben wird, genügen sollte. Hierdurch würde auch die Aufzeichnung von Verhaltensdaten zur Fahrzeugsteuerung durch den Fahrer weitgehend vermieden. Besonderer Aufmerksamkeit bedürfte allerdings auch der Übergang zwischen den von Fahrern gesteuerten und maschinell gesteuerten Fahrabschnitten. In diesem Übergangsbereich könnte eine Datenaufzeichnung von besonderer Bedeutung sein, allerdings würde es sich zugleich eindeutig um Daten handeln, die auf den Fahrer bezogen sind. Insoweit wäre in einem ersten Schritt zu klären, welcher Aufzeichnungsumfang einer vom Fahrer gesteuerten Fahrt zur Absicherung gegen nachträgliche Schutzbehauptungen erforderlich wäre.

\subsubsection{Der Stand von Wissenschaft und Technik beim autonomen Fahren}

Produkthaftungsrechtlich erscheint für den Laien der Haftungsausschluss für Fehler im Zusammenhang mit maschineller Fahrzeugsteuerung von Interesse, die nach dem Stand von Wissenschaft und Technik zum Zeitpunkt des In-Verkehr-Bringens nicht erkannt werden können. In diesem Zusammenhang des Produkthaftungsrechtes ist zunächst erneut auf die insoweit bereits in Abschn. 25.5.1 diskutierte Frage hinzuweisen, wonach im Bereich des Straßenverkehrs möglich erscheint, dass Risiken, die sich im Unfallgeschehen verwirklichen, auch auf das heutige Verkehrssystem als relevante Ursache zurückzuführen sein könnten (und nicht in allen Fällen auf das Steuerungsverhalten - von Fahrern oder Maschinen gleichermaßen). Diese Frage ist grundlegend und bedarf vorrangig der Beantwortung, um nicht auf das Vorliegen von (vermeintlichen) Produktfehlern als relevanter Ursache rückzuschließen.

Soweit der Haftungsausschlussgrund nach dem Stand von Wissenschaft und Technik aber betroffen ist, muss berücksichtigt werden, dass dieser Ausschlusstatbestand in der Praxis von ausgesprochen geringer Bedeutung ist: Ob eine Gefahr nach dem Stand von Wissenschaft und Technik nämlich tatsächlich erkennbar war, ist in zwei Schritten zu prüfen: Zunächst ist zu bestimmen, ob der Fehler für irgendeinen Wissenschaftler oder Techniker auf der Welt erkennbar war. Wenn er es war, ist die Berufung auf den Ausschlusstatbestand noch nicht ausgeschlossen, weil es dann noch auf die objektive Zugänglichkeit dieser Erkennbarkeit für den Hersteller ankommt. Ausdrücklich zu berücksichtigen sind 
dabei aber auch abweichende Meinungen einzelner Wissenschaftler, sofern diese Arbeiten den Mindestanforderungen wissenschaftlichen Arbeitens genügen [22].

Insoweit ist auch für autonome Fahrzeuge entscheidend, ob die Erkennbarkeit eines Fehlers zum Zeitpunkt des In-Verkehr-Bringens tatsächlich ausgeschlossen werden konnte (zudem müsste dies im Nachhinein, wenn es zu einem Unfallschaden gekommen ist, im zivilrechtlichen Verfahren aufgrund der dort geltenden Darlegungs- und Beweislast noch nachgewiesen werden). Nur in diesem seltenen Fall würde der Hersteller über diesen Haftungsausschlussgrund des Produkthaftungsgesetzes (und mangels Verschulden auch nach $\S 823$ Abs. 1 BGB) an Rechtssicherheit gewinnen. In der Praxis wird dieser Fall nahezu bedeutungslos sein.

Ein Bewusstsein dafür, dass Produktfehler auch nach dem Stand von Wissenschaft und Technik bei Beachtung aller notwendigen Sorgfalt möglicherweise nicht gänzlich auszuschließen sind, mithin eine „Restfehlertoleranz“ - wie sie die Norm ISO 26262 zugrunde legt - besteht, ist darum als eine technische Beschreibung des Entwicklungsprozesses aufzufassen, die im Produkthaftungsrecht keine Entsprechung findet.

\subsubsection{Mögliche Abweichungen der rechtlichen Bewertung im internationalen Kontext}

Zunächst ist darauf hinzuweisen, dass das vorliegende Kapitel von vornherein nur die Situation nach deutschem Recht in Betracht zieht und Aussagen trifft, die sich an dem in Deutschland heute geltenden Recht orientieren. Von einer Anwendung auf andere Länder kann nur eingeschränkt ausgegangen werden. Eine Übertragbarkeit der Schlussfolgerungen wird abgesehen von Zufälligkeiten insbesondere dann möglich sein, wenn es zu einer Vereinheitlichung des Rechts aufgrund internationaler Verträge (also im Rahmen des Völkerrechts) oder durch Verordnungen und Richtlinien im Rahmen der Rechtssetzung der EU gekommen ist.

Demzufolge ist darauf zu verweisen, dass hinsichtlich der dargestellten grundrechtlichen Situation ein weitgehend übereinstimmender Grundrechtsstandard - soweit vorliegend im Zusammenhang mit den deutschen Grundrechten auf Leben und körperliche Unversehrtheit von Bedeutung - beispielsweise auch durch die Charta der Grundrechte der Europäischen Union anerkannt wird. Sie weist Bezüge zur Europäischen Menschenrechtskonvention auf und mit Ausnahme des Vereinigten Königreiches und Polens wurde durch Verweis im Lissabonner Vertrag die Europäische Grundrechtscharta für die Länder der Europäischen Union für bindend erklärt. Die Rechte auf Leben und körperliche Unversehrtheit werden darin anerkannt - im Einzelnen gemäß Art. 2 Abs. 1 und Art. 3 Abs. 1 Charta der Grundrechte der Europäischen Union (vgl. [23]). Diese Betrachtung ließe sich darüber hinaus beliebig auf die Verfassungen anderer Staaten ausweiten; festzuhalten ist insoweit jedoch, dass die Grundrechtsdogmatik sich in vielen Einzelbereichen deutlich unterscheiden kann. Eine solche Untersuchung obliegt deshalb den Spezialisten der jeweiligen Rechtsmaterie. 
Das gilt gleichermaßen für das Ordnungsrecht im Straßenverkehr wie auch für das zur Anwendung kommende Haftungsrecht, das für autonomes Fahren von Bedeutung ist. Hierbei ist aber, wie in Abschn. 25.6.1 und Abschn. 25.6.2 beschrieben, bereits die Rechtslage nach deutschem Recht in sehr weiten Teilen nur sehr eingeschränkt auf das autonome Fahren übertragbar. Es erscheint durchaus möglich, dass eine vergleichbare Situation auch nach anderen Rechtsordnungen besteht. Hierfür spricht auch, dass internationale Straßenverkehrsübereinkommen wie das Wiener Übereinkommen über den Straßenverkehr von 1968 oder das Genfer Übereinkommen über den Straßenverkehr von 1949 ein mit der deutschen Straßenverkehrsordnung durchaus vergleichbares Fahrerbild sowie ständige Fahrzeugsteuerung durch einen Fahrer zugrunde legen.

Im Sinne einer besseren Vergleichbarkeit ist insoweit auf das Produkthaftungsrecht hinzuweisen, das durch die Richtlinie 85/374/EWG des Rates vom 25. Juli 1985 innerhalb der Europäischen Union bereits vereinheitlicht worden ist. Auch hier bestehen zwar neben rechtsdogmatisch zu machenden Einschränkungen noch Unsicherheiten darin, dass die Richtlinie bei ihrer Umsetzung nur hinsichtlich des Zieles verbindlich ist (ein Grundsatz, der schon nach der vormaligen Rechtslage bestand und sich heute in Art. 288 Abs. 3 des Vertrages über die Arbeitsweise der Europäischen Union findet). Dennoch liegt immerhin eine inhaltlich vergleichbare Rechtsmaterie für die innerstaatliche Rechtsanwendung vor, die nicht an ein Verschulden anknüpft (sogenannte Gefährdungshaftung).

Zusammenfassend ist deshalb festzuhalten, dass in diesen Bereichen noch erhebliche Unterschiede bestehen, die sich auf die Entwicklung autonomen Fahrens auswirken könnten. Das ist aber aufgrund der nationalen Prägung des Rechts nicht im Einzelnen absehbar und - soweit gegenwärtig ersichtlich - weiterhin untersuchungsbedürftig.

\subsubsection{Sonderfrage: Aufsichtspflicht über Insassen autonomer Fahrzeuge}

Der Nutzen autonomen Fahrens liegt u. a. darin, dass Aufmerksamkeitsressourcen des Menschen nicht mehr für die Ausführung der Fahraufgabe benötigt werden. Dies kann potenziell dazu führen, die Anwesenheit des „Fahrers“ und damit der aufsichtspflichtigen Person im Fahrzeug insgesamt infrage zu stellen. So würde ein erheblicher Mehrnutzen autonomen Fahrens sich daraus ergeben, dass auch Mobilitätswünsche von Kindern - ohne eines Fahrers zu bedürfen - erfüllt werden könnten. Es ist deshalb keineswegs auszuschließen, dass hieraus Gefahren resultieren, wenn es sich - wie voraussichtlich häufig - beim Fahrer um die aufsichtspflichtige Person handelt, die dann nicht mehr anwesend ist.

Allerdings erscheint von den vorliegenden Stellvertreterapplikationen nur der Fall des „Vehicle-on-Demand“ überhaupt geeignet, eine solche Transportaufgabe ohne die Begleitung Aufsichtspflichtiger überhaupt durchzuführen: Selbst die Stellvertreterapplikation des „Vollautomaten mit Verfügbarkeitsfahrer“ setzt die Anwesenheit eines Fahrers voraus, um nicht freigegebene Verkehrsbereiche oder vom autonomen Fahren ausgenommene Streckenabschnitte befahren zu können (s. Kap. 2). Da die Stellvertreterapplikation des 
„Vehicle-on-Demand“ schon keinen Fahrerplatz aufweist (sondern einen völlig frei gestalteten Innenraum), erscheinen Gefahren und Auswirkungen aus der Nutzung durch aufsichtsbedürftige Personen sich zunächst auf den Fahrzeuginnenraum zu beschränken und sich nicht auf den Straßenverkehr auszuwirken. Dann würde sich die Frage der Abwesenheit aufsichtspflichtiger Personen allerdings nicht mit Relevanz im Bereich des Straßenverkehrs stellen. Es erscheint allerdings ebenso denkbar, dass entsprechende autonome Fahrzeuge noch einen Fahrerplatz aufweisen. Dann wäre aber auch potenziell ein erheblicher negativer Einfluss auf die Fahrzeugsteuerung möglich, indem eine Übersteuerung der autonomen Fahrfunktion über die Bedienelemente erfolgt.

Es bleibt festzustellen, dass die rechtliche Frage der Aufsichtspflicht nicht von der Einführung autonomen Fahrens abhängig ist. Vielmehr können schon heute eigenständige Schadensersatzpflichten durch eine Aufsichtspflichtverletzung herbeigeführt werden (vgl. § 832 Bürgerliches Gesetzbuch (BGB)). So besteht eine gesetzliche Aufsichtspflicht der Eltern im Rahmen elterlicher Sorge, $\S \S 1626$ ff. BGB. Damit wäre abhängig von Alter, Eigenart, Charakter, Kenntnissen und Fähigkeiten beispielsweise eines Kindes zu bestimmen, welche Intensität der Aufsicht angesichts einer Voraussehbarkeit schädigenden Verhaltens im Einzelfall geboten ist [24]. Wird eine sich ergebende Pflicht verletzt und kommt es hierdurch zu einem Schaden, kann sich ein eigenständiger Schadensersatzanspruch (neben einem gegebenenfalls gegen das Kind selbst bestehenden Anspruch) auch gegenüber den Eltern des Kindes ergeben. Insoweit könnte aus den entsprechenden Möglichkeiten autonomer Fahrzeuge resultieren, dass der korrekten Erfüllung von Aufsichtspflichten in diesem Zusammenhang eine neue, bislang unbekannte Bedeutung zukommt. Ein rechtlicher Änderungsbedarf ist aber nicht ersichtlich.

\subsection{Fazit}

Aus der mit dem vorliegendem Kapitel vorgenommenen Betrachtung autonomen Fahrens aus einer rechtlichen Perspektive tritt - neben den bereits in den jeweiligen Unterabschnitten aufgeworfenen Fragen - vor allem ein grundlegender Aspekt in Erscheinung, der im Zusammenhang mit dem Wandel von einer menschlichen zu einer maschinellen Fahrzeugsteuerung möglicherweise deutlicher als bisher erkennbar wird: Auch wenn heute davon ausgegangen wird, dass grundsätzlich jeder Unfall von Kraftfahrzeugen im Straßenverkehr auf ein Versagen bei der Steuerung zurückzuführen ist, muss im Sinne einer naturwissenschaftlichen Aufarbeitung danach gefragt werden, inwieweit das heutige Verkehrssystem angesichts der dort herrschenden Rahmenbedingungen nicht eine eigenständige, relevante Ursache für einen Teil des heutigen Unfallgeschehens darstellt. Die Beantwortung dieser Frage ist dabei nicht Selbstzweck, sondern kann helfen zu erkennen, ob und gegebenenfalls welche Veränderungen bei der Fahrzeugsteuerung unter bestimmten Umgebungsbedingungen geeignet sind, Unfälle zu vermeiden. Für die Gestaltung eines autonomen Fahrzeuges als ein sicheres Produkt ist dies seitens eines Herstellers zudem entscheidend hinsichtlich der Ausgestaltung autonomer maschineller Steuerungsfunktionen. 
Weiterhin scheint für die weitere Bearbeitung - wie auch für die Rechtssicherheit sowohl der (Fahrzeug-)Betriebsverantwortlichen als auch der Hersteller autonomer Fahrzeuge - die Beschreibung maschineller Steuerungsqualität im Sinne einer Leistungsdefinition ein wichtiger Meilenstein zu sein. Hierauf könnte eine Bewertung aufbauen, die ermittelt, genau in welchen Bereichen beispielsweise bezüglich der Kommunikationsfähigkeiten autonomer Fahrzeuge oder Fehlerkompensationsfähigkeiten noch Entwicklungsbedarf besteht. Umgekehrt wird eine Betrachtung ermöglicht, wie der entsprechende rechtliche Rahmen ausgestaltet sein muss, um zu einem funktionierenden „Verkehrssystem Straße“ unter Beteiligung autonomer Fahrzeuge zu kommen.

\section{Literatur}

1. Homann, K.: Wirtschaft und gesellschaftliche Akzeptanz: Fahrerassistenzsysteme auf dem Prüfstand. In Maurer, M.; Stiller, C. (Hrsg.), Fahrerassistenzsysteme mit maschineller Wahrnehmung. Berlin (2005)

2. Projekt: Realisierung einer automatisierten U-Bahn in Nürnberg (RUBIN) - Automatisierung der U-Bahn-Linien U2 und U3 in Nürnberg, ausgeführt durch die Siemens Mobility (ehemals: Siemens TS). Konzeptionelle Darstellung inzwischen nur noch unter: https://de.wikipedia.org/ wiki/RUBIN sowie Hinweis auf das Pilotprojekt der Siemens Mobility unter: http://www. siemens.de/staedte/referenzprojekte/seiten/rubin_nuernberg.aspx

3. Murswiek, Dietrich: Grundgesetz Kommentar (zu Art. 2 GG). In Sachs, M. (Hrsg.), 6. Auflage, München (2011)

4. Heldmann, Horst: 15 Jahre Strafbewehrung der Gurtanlegepflicht in Zeitschrift für Verkehrssicherheit (ZVS) 45 (1999), S. 146-159

5. Statistisches Bundesamt, Verkehrsunfälle 2013 - Fachserie 8, Reihe 7. Wiesbaden (2014)

6. Herber, Franz-Rudolf: Die öffentliche Straße als öffentliche Sache-öffentliche Sachherrschaft und private Sachherrschaft. In Kodal, K. (Hrsg.): Straßenverkehrsrecht. 7. Auflage, München (2010)

7. Stahlhut, Ulrich: Der schlichte Gemeingebrauch. In Kodal, K. (Hrsg.): Straßenverkehrsrecht. 7. Auflage, München (2010)

8. Daimler AG: Mercedes Benz, Betriebsanleitung Interaktiv S-Klasse. Menüpunkte: „Vertiefen“/ „Fahrsysteme“/ „DISTRONIC PLUS“. Online abgerufen: http://www4.mercedes-benz.com/ manual-cars/ba/cars/221/de/manual_base.shtml (am 04.08.2014)

9. Maurer, Markus: Entwurf und Test von Fahrerassistenzsystemen. In Winner, H.; Hakuli, S. und Wolf, G. (Hrsg.), Handbuch Fahrerassistenzsysteme. 2. Auflage, Wiesbaden (2012)

10. Gasser, Tom M.; Arzt, Clemens; Ayoubi, Mihiar; Bartels, Arne; Bürkle, Lutz; Eier, Jana; Flemisch, Frank; Häcker, Dirk; Hesse, Tobias; Huber, Werner; Lotz, Christine; Maurer, Markus; Ruth-Schumacher, Simone; Schwarz, Jürgen; Vogt, Wolfgang: Rechtsfolgen zunehmender Fahrzeugautomatisierung. Gemeinsamer Schlussbericht der BASt-Projektgruppe „Rechtsfolgen zunehmender Fahrzeugautomatisierung“ Dokumentteil 1. Wirtschaftsverlag NW, Bergisch Gladbach, 2012 (Heft F 83)

11. Bundesverfassungsgerichtsentscheidung Band 47, Seiten 46 - 85 (Sexualkundeunterricht), Ausführungen zum sogenannten „Wesentlichkeitsgrundsatz“: Seite 79. Beschluss des 1. Senates v. 21. Dez. 1977

12. Bundesverfassungsgerichtsentscheidung Band 83, Seiten 130-155 (Josefine Mutzenbacher), Ausführungen zum sogenannten „Wesentlichkeitsgrundsatz“: Seite 142. Beschluss des 1. Senates v. 27. Nov. 1990 
13. Bundesverfassungsgerichtsentscheidung Band 49, Seiten 89-147 (Kalkar I), Ausführungen zum „Parlamentsvorbehalt“: Seiten 124ff. Beschluss des 2. Senates v. 08. August 1978

14. König, Peter: Kommentierung zur Straßenverkehrsordnung und Straßenverkehrsgesetz. In Hentschel, P.; König, P.; Dauer, P.: Straßenverkehrsrecht (Kommentar). 42. Auflage, München (2013)

15. Spiegel, Richard: Die neuen Erkenntnisse über die Reaktionszeit des Kraftfahrers und die Rechtsprechung. Veröffentlichung der auf dem 20. Verkehrsgerichtstag am 28. und 29. Januar 1982 gehaltenen Referate, Arbeitskreis I. Hamburg (1982)

16. Seiniger, Patrick; Bartels Oliver; Pastor, Claus; Wisch, Marcus: An Open Simulation Approach to Identify Chances and Limitations for Vulnerable Road User (VRU) Active Safety. Traffic Injury and Prevention, Heft 14, S. 2-12 (2013)

17. Albrecht, Frank: Fahrerassistenzsysteme zur Geschwindigkeitsbeeinflussung. Deutsches Autorecht (DAR), Heft 4, S. 186-198 (2005)

18. Bahr, Michael; Sturzbecher, Dietmar: Bewertungsgrundlagen zur Beurteilung der Fahrbefähigung bei der praktischen Fahrerlaubnisprüfung. Beitrag im Rahmen des 6. Darmstädter Kolloquiums: Maßstäbe des sicheren Fahrens (2013)

19. Merten, Klaus: Informelle Zeichengebung im Straßenverkehr. Bericht der Bundesanstalt für Straßenwesen Nr. 53. Köln (1981)

20. Merten, Klaus: Kommunikationsprozesse im Straßenverkehr. Unfall- und Sicherheitsforschung im Straßenverkehr, Heft 14, S. 115-126. Bonn (1977)

21. Beschluss des Oberlandesgerichtes Hamm v. 30.10 .1995 (Aktenzeichen: 2 Ss OWi 1097/95) in Verkehrsrechtssammlung Band 91, Nr. 50, Seite 125 ff. (1996)

22. Wagner, Gerhard: Bearbeiter der Kommentierung des Produkthaftungsgesetzes und $\S 823$ Abs. 1 BGB in Münchener Kommentar zum BGB, Band 5, Schuldrecht Besonderer Teil III, $\S \S 705-853$ BGB, Partnerschaftsgesellschaftsgesetz, Produkthaftungsgesetz. 5. Auflage, München (2009)

23. Charta der Grundrechte der Europäischen Union. Amtsblatt der Europäischen Union C 83/ 389 vom 30.03.2010 (2010/C 83/02) (2010). Im Internet verfügbar unter: http://eur-lex.europa.eu/ LexUriServ/LexUriServ.do?uri=OJ:C:2010:083:0389:0403:DE:PDF (Abruf am 22.08.2014)

24. Sprau, Hartwig: Kommentierung zu $\S \S 631-853$ des Bürgerlichen Gesetzbuches. In Palandt, Bürgerliches Gesetzbuch, Kommentar, 73. Auflage, München (2014) 